\title{
Potential Theory on Minimal Hypersurfaces II: Hardy Structures and Schrödinger Operators
}

\author{
Joachim Lohkamp ${ }^{1}$
}

Received: 26 May 2018 / Accepted: 28 July 2020 / Published online: 18 August 2020

(C) The Author(s) 2020

\begin{abstract}
Area minimizing hypersurfaces and, more generally, almost minimizing hypersurfaces frequently occur in geometry, dynamics and physics. A central problem is that a general (almost) minimizing hypersurface $H$ contains a complicated singular set $\Sigma$. Nevertheless, we manage to develop a detailed potential theory on $H \backslash \Sigma$ applicable to large classes of linear elliptic second order operators. We even get a fine control over their analysis near $\Sigma$. This is Part 2 of this two parts work.
\end{abstract}

Keywords Potential theory: boundary Harnack inequalities - Minimal hypersurfaces . Singularities · Uniform spaces · Gromov hyperbolicity $\cdot$ Schrödinger operators

Mathematics Subject Classification (2010) 30L10 - 31C12 · 49Q15 · 51M10 · 53A10 · 53A30

\section{Introduction}

In this paper, we further develop the potential theory on generally singular area minimizing and, more generally, on almost minimizing hypersurfaces we initiated in the first part of this work [17]. We focus on elliptic operators naturally associated to such hypersurfaces, for instance, from the minimality constraint of the hypersurface and the geometry of the ambient space. This analysis can be applied to investigate the ambient space. A famous example hereof is the (non)existence of positive scalar curvature metrics on compact manifolds which is closely related to the potential theory of the conformal Laplacian on its minimal hypersurfaces $[16,21]$.

We know from [18] that the $\mathcal{S}$-uniformity of $H \backslash \Sigma$, for an (almost) minimizing hypersurface $H$ with singular set $\Sigma$, suffices to establish a transparent potential theory on $H \backslash \Sigma$. This comes along with boundary Harnack inequalities on $H \backslash \Sigma$ relative to the boundary $\Sigma$ and a Martin theory saying that $\Sigma$ is just the Martin boundary for a large class of operators, the so-called $\mathcal{S}$-adapted operators.

Joachim Lohkamp

j.lohkamp@uni-muenster.de

1 Mathematisches Institut, Universität Münster, Einsteinstrasse 62, Münster, Germany 
In the present paper, we take the applications of $\mathcal{S}$-structures to the elliptic analysis on (almost) minimizers much further. We first show that (almost) minimizers actually carry an enhanced version of $\mathcal{S}$-structures, namely a so-called Hardy $\mathcal{S}$-structure. These satisfy an additional geometric coupling relation between $\mathcal{S}$-structures and the curvature of almost minimizers. It follows that many classical elliptic operators, like the Jacobi field operator or the conformal Laplacian, are actually $\mathcal{S}$-adapted so that our techniques from [17] apply. Typically, these operators will be Schrödinger operators $L=L(H)$ on $H \backslash \Sigma$, and the assignment $H \mapsto L(H)$ commutes with blow-ups. That is, for infinite scalings $m \cdot H$ around any $p \in \Sigma \subset H, L(m \cdot H)$ converges to $L(C)$ on any tangent cone $C$ in $p$ as $m \rightarrow \infty$. For these so-called natural Schrödinger operators our theory works particularly nicely.

The question of understanding their ground states and Green's functions is of great importance. Towards the singular set these characteristic solutions all have minimal growth compared with other solutions. This property is the starting point for our further analysis. Namely, we prove the allegedly plausible but rather non-trivial fact that minimal growth of solutions $u>0$ on $H \backslash \Sigma$ of $L(H) \phi=0$ towards some singular point $p \in \Sigma$ persists under blow-ups.

In particular, we find that, for any tangent cone $C$ around $p$, solutions of $L(C) \phi=0$ that are induced from $u$ have minimal growth towards the singularity set $\Sigma_{C}$ of the cone. The Martin theory in [17] shows that these solutions are unique up to constant multiples and they admit a separation of variables. From this observation we can start a scheme of inductive descent and continue with blow-ups in singular points of $C \backslash\{0\}$ to get simpler cones of the form $C^{*} \times \mathbb{R}$ until we reach $C^{\circ} \times \mathbb{R}^{k}$, where $C^{\circ}$ is only singular at its tip. The resulting tree of blow-ups together with the induced solutions, analyzed using the individual potential theory on these blow-ups, allows a detailed analysis of solutions on $H \backslash \Sigma$ near $\Sigma$.

As in [18], this analysis does not use the structure of $\Sigma$ itself. Instead it is naturally and tightly connected to $\mathcal{S}$-structures and the properties of the associated hyperbolic unfoldings.

\subsection{Basic Concepts and Notations}

We collect some basic notations, cf. [17, Chapter 1.1], [18, Appendix A] and also Ch. 3.1 for details.

In the following, $H^{n}$ denotes an integer multiplicity rectifiable current of dimension $n \geq$ 2 with connected support inside some complete, smooth Riemannian manifold $\left(M^{n+1}, g_{M}\right)$. By $\Sigma_{H}$ (or simply $\Sigma$ if there is no risk of confusion) we denote the set of singular points of $H$. Throughout this paper, $H^{n}$ will be either an area minimizer or, more generally, an almost (area) minimizer. These notions are properly defined and discussed in Ch. 3.1 below.

For now, we note that in all these cases it is known that $\Sigma_{H}$ has Hausdorff dimension $\leq \operatorname{dim} H-7$. We also write $\sigma_{C}$, in place of $\Sigma_{C}$, for the singular set of a minimal cone $C$, in particular if we want to emphasize that $C$ is viewed as a tangent cone.

The induced Riemannian metric on $H \backslash \Sigma$ will be denoted by $g_{H}$. For $\lambda>0$, we let $\lambda \cdot M$ or $\lambda \cdot H \backslash \Sigma$ denote the conformally rescaled Riemannian manifolds $\left(M, \lambda^{2} \cdot g\right)$ or $\left(H \backslash \Sigma, \lambda^{2} \cdot g_{H}\right)$. We refer to the induced distance function $d=d_{H}$ on $H \backslash \Sigma$ as intrinsic distance. Further, $A=A_{H}$ is the second fundamental form of $H \backslash \Sigma \subset M$ and $\left|A_{H}\right|$ its norm.

Basic Classes of such currents.

$\mathcal{H}_{n}^{c}: \quad H^{n} \subset M^{n+1}$ is compact locally mass minimizing without boundary. 
$\mathcal{H}_{n}^{\mathbb{R}}: \quad H^{n} \subset \mathbb{R}^{n+1}$ is a complete hypersurface in flat Euclidean space $\left(\mathbb{R}^{n+1}, g_{\text {eucl }}\right)$ with

$0 \in H$ that is an oriented minimal boundary of some open set in $\mathbb{R}^{n+1}$.

$\mathcal{H}_{n}: \quad \mathcal{H}_{n}:=\mathcal{H}_{n}^{c} \cup \mathcal{H}_{n}^{\mathbb{R}}$ and $\mathcal{H}:=\bigcup_{n \geq 1} \mathcal{H}_{n}$. We briefly refer to $H \in \mathcal{H}$ as an area minimizer.

$\mathcal{C}_{n}: \quad \mathcal{C}_{n} \subset \mathcal{H}_{n}^{\mathbb{R}}$ is the space of area minimizing $n$-cones in $\mathbb{R}^{n+1}$ with tip in 0 .

$\mathcal{S C}_{n}: \mathcal{S C}_{n} \subset \mathcal{C}_{n}$ is the subset of cones which are at least singular in 0 .

$\mathcal{K}_{n-1}$ : For any area minimizing cone $C \subset \mathbb{R}^{n+1}$ with tip 0 , we get the non-minimizing minimal hypersurface $S_{C}$ in the unit sphere

$$
S_{C}:=\partial B_{1}(0) \cap C \subset S^{n} \subset \mathbb{R}^{n+1}
$$

and we set $\mathcal{K}_{n-1}:=\left\{S_{C} \mid C \in \mathcal{C}_{n}\right\}$. We write $\mathcal{K}=\bigcup_{n \geq 1} \mathcal{K}_{n-1}$ for the space of all such hypersurfaces $S_{C}$.

$\mathcal{G}_{n}^{c}$ : $\quad H^{n} \subset M^{n+1}$ is a compact almost minimizer. In this series of papers this means it is a compact almost minimizing oriented boundary cf. Def. 3.5 for details. We set $\mathcal{G}^{c}:=$ $\bigcup_{n \geq 1} \mathcal{G}_{n}^{c}$.

$\mathcal{G}_{n}: \quad \mathcal{G}_{n}:=\mathcal{G}_{n}^{c} \cup \mathcal{H}_{n}^{\mathbb{R}}$ and $\mathcal{G}:=\bigcup_{n \geq 1} \mathcal{G}_{n}$.

We denote the one-point compactification of a hypersurface $H \in \mathcal{H}_{n}^{\mathbb{R}}$ by $\widehat{H}$. For the singular set $\Sigma_{H}$ of some $H \in \mathcal{H}_{n}^{\mathbb{R}}$ we always add $\infty_{H}$ to $\Sigma$ as well, even when $\Sigma$ is already compact, to define $\widehat{\Sigma}_{H}:=\Sigma_{H} \cup \infty_{H}$. On the other hand, for $H \in \mathcal{G}_{n}^{c}$ we set $\widehat{H}=H$ and $\widehat{\Sigma}=\Sigma$.

We call an assignment $\langle A\rangle$ which associates with any $H \in \mathcal{G}$ a function $\langle A\rangle_{H}: H \backslash$ $\Sigma_{H} \rightarrow \mathbb{R}$ an $\mathcal{S}$-transform provided it satisfies the subsequent list of axioms.

(S1) Trivial Gauge If $H \subset M$ is totally geodesic, then $\langle A\rangle_{H} \equiv 0$.

(S2) $\mathcal{S}$-Properties If $H$ is not totally geodesic, then the level sets $\mathbb{A}_{c}:=\langle A\rangle_{H}^{-1}(c)$, for $c>0$, which we call the $|A|$-skins, surround the level sets of $|A|$ :

$$
\langle A\rangle_{H}>0,\langle A\rangle_{H} \geq\left|A_{H}\right| \text { and }\langle A\rangle_{H}(x) \rightarrow \infty \text {, for } x \rightarrow p \in \Sigma_{H} .
$$

Like $\left|A_{H}\right|,\langle A\rangle_{H}$ anticommutes with scalings, i.e., $\langle A\rangle_{\lambda \cdot H} \equiv \lambda^{-1} \cdot\langle A\rangle_{H}$ for any $\lambda>0$.

(S3) Lipschitz regularity If $H$ is not totally geodesic, and thus $\langle A\rangle_{H}>0$, we define the $\mathcal{S}$-distance $\delta_{\langle A\rangle_{H}}:=1 /\langle A\rangle_{H}$. This function is $L_{\langle A\rangle}$-Lipschitz regular for some constant $L_{\langle A\rangle}=L(\langle A\rangle, n)>0$, i.e.,

$$
\left|\delta_{\langle A\rangle_{H}}(p)-\delta_{\langle A\rangle_{H}}(q)\right| \leq L_{\langle A\rangle} \cdot d_{H}(p, q) \text { for any } p, q \in H \backslash \Sigma \text { and any } H \in \mathcal{G}_{n} .
$$

If $H$ is totally geodesic, and thus $\langle A\rangle_{H} \equiv 0$, we set $\delta_{\langle A\rangle_{H}} \equiv \infty$ and $\mid \delta_{\langle A\rangle_{H}}(p)-$ $\delta_{\langle A\rangle_{H}}(q) \mid \equiv 0$.

(S4) Naturality If $H_{i} \in \mathcal{H}_{n}, i \geq 1$, is a sequence converging to the limit space $H_{\infty} \in$ $\mathcal{H}_{n}$, then $\langle A\rangle_{H_{i}} \stackrel{C^{\alpha}}{\longrightarrow}\langle A\rangle_{H_{\infty}}$ for any $\alpha \in(0,1)$. For general $H \in \mathcal{G}_{n}$, this holds for blow-ups: $\langle A\rangle_{\tau_{i} \cdot H} \stackrel{C^{\alpha}}{\longrightarrow}\langle A\rangle_{H_{\infty}}$, for any sequence $\tau_{i} \rightarrow \infty$ so that $\tau_{i} \cdot H \rightarrow H_{\infty} \in \mathcal{H}_{n}^{\mathbb{R}}$.

Remark 1.1 (i) A construction of an $\mathcal{S}$-transform by merging the metric $g_{H}$ on $H \backslash \Sigma_{H}$ and the second fundamental form $A=A_{H}$ into one scalar function $\langle A\rangle_{H}$ on $H \backslash \Sigma_{H}$ was given in [18].

(ii) The $\mathcal{S}$-distance $\delta_{\langle A\rangle}$ is merely Lipschitz regular, but admits a Whitney type smoothing $\delta_{\langle A\rangle^{*}}$ which satisfies $(\mathrm{S} 1)-(\mathrm{S} 3)$ and which is quasi-natural in the sense that 
$c_{1} \cdot \delta_{\langle A\rangle}(x) \leq \delta_{\langle A\rangle}(x) \leq c_{2} \cdot \delta_{\langle A\rangle}(x)$ for some constants $c_{1}, c_{2}>0$, cf. [18, Proposition B.3] for details.

\subsection{Statement of Results}

In [17] we introduced $\mathcal{S}$-adapted operators and studied their basic potential theory on almost minimizers $H \in \mathcal{G}$. This class of operators is rich but a priori unrelated to the geometry of $\left(H \backslash \Sigma, g_{H}\right)$. Further, it depends on the chosen $\mathcal{S}$-transform $\langle A\rangle$. In the first part of this paper we resolve this (apparent) issue. We show that there are $\mathcal{S}$-transforms with a tighter coupling between $|A|$ and $\langle A\rangle$ beyond the axioms (S1)-(S4). Then we use this to prove that many classical operators are $\mathcal{S}$-adapted. Typically, these operators are symmetric and we are mostly interested in eigenvalue problems, so we shall focus on this situation.

Shifted $\mathcal{S}$-Adapted Operators We recall the following definitions and results from [17, Definition 1.12, Theorem $7(\mathcal{S}$-Hardy Inequality)]. For this we use special charts for $H \backslash \Sigma$, namely $\mathcal{S}$-adapted charts. These are bi-Lipschitz charts $\psi_{p}: B_{R}(p) \rightarrow \mathbb{R}^{n}$ centered in $p \in H \backslash \Sigma$, for some Lipschitz constant $K$ independent of $p$, and where the radius $R$ of the ball is $\Theta(p):=\Gamma /\langle A\rangle(p)$ for some $\Gamma(H, K,\langle A\rangle)>0$, cf. [17, Chapter 2.3] and [18, Proposition B.1].

Definition 1.2 Let $H \in \mathcal{G}$. We call a symmetric second order elliptic operator $L$ on $H \backslash \Sigma$ shifted $\mathcal{S}$-adapted supposed the following two conditions hold:

$\langle$ A $\rangle$-Adaptedness $L$ satisfies $\mathcal{S}$-weighted uniformity conditions with respect to the charts $\psi_{p}$. Namely, we can write

$$
-L(u)=\sum_{i, j} a_{i j} \cdot \frac{\partial^{2} u}{\partial x_{i} \partial x_{j}}+\sum_{i} b_{i} \cdot \frac{\partial u}{\partial x_{i}}+c \cdot u,
$$

for some $a_{i j} \in C^{1}(H \backslash \Sigma)$ and $b_{i}, c \in C^{\alpha}(H \backslash \Sigma)$, for some $\alpha \in(0,1)$ and any $i, j$. Moreover, we assume there exists a $k_{L}=k \geq 1$ and some $\beta \in(0,1]$ such that for all $p \in H \backslash \Sigma, \xi \in \mathbb{R}^{n}$, we have

$$
k^{-1} \cdot \sum_{i} \xi_{i}^{2} \leq \sum_{i, j} a_{i j}(p) \cdot \xi_{i} \xi_{j} \leq k \cdot \sum_{i} \xi_{i}^{2},
$$

$\delta_{\langle A\rangle}^{\beta}(p) \cdot\left|a_{i j}\right|_{C^{\beta}\left(B_{\Theta(p)}(p)\right)} \leq k, \delta_{\langle A\rangle}(p) \cdot\left|b_{i}\right|_{L^{\infty}\left(B_{\Theta(p)}(p)\right)} \leq k$ and $\delta_{\langle A\rangle}^{2}(p) \cdot|c|_{L^{\infty}\left(B_{\Theta(p)}(p)\right)} \leq k$.

$\langle$ A $\rangle$-Finiteness There exists a finite constant $\tau=\tau(L,\langle A\rangle, H)>-\infty$ such that for any $C^{2}$-function $f$ which is compactly supported in $H \backslash \Sigma$, we have

$$
\int_{H} f \cdot L f d V \geq \tau \cdot \int_{H}\langle A\rangle^{2} \cdot f^{2} d V .
$$

Remark 1.3 In [18] the operators in this definition are called $\langle A\rangle^{\times}$-adapted and (shifted) $\mathcal{S}^{\times}$-adapted for visual distinction between them and more general not necessarily symmetric and less regular operators. However, in this paper we exclusively consider the symmetric cases described above and thus we can safely call them $\langle A\rangle$-adapted and (shifted) $\mathcal{S}$-adapted. 
Definition 1.4 The largest $\tau \in(-\infty,+\infty)$ such that Eq. 1 holds is the principal eigenvalue $\lambda_{L, H}^{\langle A\rangle}$ of $\delta_{\langle A\rangle}^{2} \cdot L$. The operator $L$ is called $\mathcal{S}$-adapted if $\lambda_{L, H}^{\langle A\rangle}>0$.

For any shifted $\mathcal{S}$-adapted $L$ we set

$$
L_{\lambda}:=L-\lambda \cdot\langle A\rangle^{2} \cdot I d, \text { for } \lambda \in \mathbb{R} .
$$

Again, $L_{\lambda}$ is shifted $\mathcal{S}$-adapted. Moreover, it is $\mathcal{S}$-adapted if and only if $\lambda<\lambda_{L, H}^{\langle A\rangle}$.

Hardy Relations Coming back to the inequality $\langle A\rangle_{H} \geq\left|A_{H}\right|$, we note that there cannot be a pointwise inverse inequality. For instance, singular cones may contain subcones where $|A| \equiv 0$ whereas $\langle A\rangle>0$. However, we can prove an inverse integral inequality for special $\mathcal{S}$-transforms. To exclude the previous counterexample we need to include a gradient into the integrals. We obtain thus a generalization of both the Poincaré inequality and the sharper Hardy inequality for the Laplacian $-\Delta_{E u c l}$ on flat Euclidean domains $D \subset \mathbb{R}^{n}$, to operators which couple to $|A|$ on curved manifolds $H \backslash \Sigma$ with boundary $\Sigma, H \in \mathcal{G}$, cf. Ch. 2.1.

Theorem 1 (Hardy $\mathcal{S}$-Structures, see Theorem 2.1) There are $\mathcal{S}$-transforms $\langle A\rangle$ such that for all $H \in \mathcal{G}$ and $f \in C_{0}^{2}(H \backslash \Sigma)$, the space of $C^{2}$-functions compactly supported in $H \backslash \Sigma$, the following Hardy relations hold:

$\left(H_{c}\right)$ For any compact $H \in \mathcal{G}^{c}$ and any $C^{\alpha}$-regular $(2,0)$-tensor $B, \alpha \in(0,1)$, on the ambient space $M$ of $H$ with $\left.B\right|_{H} \not \equiv-A_{H}$ there exists a constant $k_{H ; B}>0$, called Hardy constant, such that

$$
\int_{H}|\nabla f|^{2}+\left.|A+B|_{H}\right|^{2} \cdot f^{2} d V \geq k_{H ; B} \cdot \int_{H}\langle A\rangle^{2} \cdot f^{2} d V \geq \frac{k_{H ; B}}{L_{\langle A\rangle}^{2}} \cdot \int_{H} \frac{f^{2}}{\operatorname{dist}_{H}(x, \Sigma)^{2}} d V .
$$

For singular $H \in \mathcal{G}^{c},|A|$ is unbounded whereas $|B|_{H} \mid$ remains bounded, hence, in this case the condition $\left.B\right|_{H} \not \equiv-A$ is redundant.

$\left(H_{\mathbb{R}}\right) \quad$ For $H \in \mathcal{H}_{n}^{\mathbb{R}}$ we only consider the case $B=0$. Then the Hardy constant depends solely on the dimension, that is, $k_{H ; 0}=k_{n}>0$, and we have

$$
\int_{H}|\nabla f|^{2}+|A|^{2} \cdot f^{2} d V \geq k_{n} \cdot \int_{H}\langle A\rangle^{2} \cdot f^{2} d V \geq \frac{k_{n}}{L_{\langle A\rangle}^{2}} \cdot \int_{H} \frac{f^{2}}{\operatorname{dist}_{H}(x, \Sigma)^{2}} d V .
$$

These relations also apply to the case $\Sigma=\emptyset$ via the convention $1 / \operatorname{dist}_{H}(x, \Sigma)=0$. An $\mathcal{S}$-transform satisfying both axioms $(H)=\left(H_{c}\right)+\left(H_{\mathbb{R}}\right)$ is called a Hardy $\mathcal{S}$-transform.

Remark 1.5 (i) $\operatorname{dist}_{H}(x, \Sigma)$ denotes the intrinsic distance of $x \in H \backslash \Sigma$ to $\Sigma$. It is a not quite obvious fact, which follows from the uniformity properties of $H \backslash \Sigma$, that this is a well-controlled finite number [18, Chapter 2.3]. There is even a constant $c_{n}^{\mathbb{R}} \in(0,1)$ depending only on the dimension $n$ such that for any $H \in \mathcal{H}_{n}^{\mathbb{R}}$ and $p, q \in H \subset \mathbb{R}^{n+1}$ the extrinsic and the intrinsic distances are equivalent [18, 2.10]:

$$
c_{n}^{\mathbb{R}} \cdot d_{g_{H}}(p, q) \leq d_{g_{\mathbb{R}^{n+1}}}(p, q) \leq d_{g_{H}}(p, q) .
$$

(ii) The ambient field $B$ incorporates geometric or physical constraints on $M$. An example is the second fundamental form of $M$ in a still higher dimensional space. In this context, we consider hypersurfaces in $\mathcal{H}_{n}^{\mathbb{R}}$ primarily as limit spaces under blow-ups, that is, infinite scaling of a given $H \in \mathcal{G}$ around its singularities. Since $|B|_{\lambda M}=$ 
$\lambda^{-1} \cdot|B|_{M}$, for $\lambda>0$, and $\lambda \rightarrow \infty$ during the blow-up process, this suggests to focus on the case $B=0$ on $H \in \mathcal{H}_{n}^{\mathbb{R}}$.

(iii) $\left(H_{c}\right)$ implies the Poincaré type inequality $\int_{H}|\nabla f|^{2}+\left.|A+B|_{H}\right|^{2} \cdot f^{2} d V \geq k_{H ; B}^{*}$. $\int_{H} f^{2} d V$ for some $k_{H ; B}^{*}>0$ since $\langle A\rangle$ remains positively lower bounded when $H$ is compact.

Henceforth, we only use $\mathcal{S}$-transforms on $\mathcal{G}$ that are Hardy. This allows us to show that many operators which occur in the Euler-Lagrange equations of natural variational integrals on $H$ are actually (shifted) $\mathcal{S}$-adapted. We have the following list of basic examples, where scal $_{H}$ and scal $_{M}$ denote the scalar curvature of $H$ and $M$ and $\operatorname{Ric}_{M}$ the Ricci curvature of $M$.

Theorem 2 (Curvature Constraints, see Theorem 2.8) Let $\langle A\rangle$ be a Hardy $\mathcal{S}$-transform. Further, let $H=H^{n} \in \mathcal{G}_{n}$ with $H^{n} \subset M^{n+1}$ and such that $H \backslash \Sigma$ is non-totally geodesic. Then we have on $H \backslash \Sigma$ :

(i) The conformal Laplacian $L_{H}:=-\Delta_{H}+\frac{n-2}{4(n-1)} \cdot s c a l_{H}$ is shifted $\mathcal{S}$-adapted. If, in addition, scal $M \geq 0$ and $H \in \mathcal{H}$, then $L_{H}$ is even $\mathcal{S}$-adapted.

(ii) More generally, let $S$ be any bounded $C^{\alpha}$-function on $M$. Then the $S$-conformal Laplacian $L_{H, S}:=-\Delta_{H}+\frac{n-2}{4(n-1)} \cdot\left(\operatorname{scal}_{H}-\left.S\right|_{H}\right)$ is shifted $\mathcal{S}$-adapted. If, in addition, scal $_{M} \geq S$ and $H \in \mathcal{H}$, then $L_{H, S}$ is even $\mathcal{S}$-adapted.

(iii) The Laplacian $-\Delta_{H}$ is shifted $\mathcal{S}$-adapted. For general $H \in \mathcal{G}$, the principal eigenvalue is non-negative $\lambda_{-\Delta, H}^{\langle A\rangle} \geq 0$. For $H \in \mathcal{G}^{c}$, the principal eigenvalue $\lambda_{-\Delta, H}^{\langle A\rangle}$ vanishes and the ground state is given by a constant function and $H \backslash \Sigma$ has the Liouville property saying that all bounded harmonic functions are constant.

(i) For any $C^{\alpha}$-regular (2,0)-tensor $B$ on the ambient space $M$, with $\left.B\right|_{H} \not \equiv-A$ if $H \in \mathcal{G}^{c}$ and with $B=0$ if $H \in \mathcal{H}_{n}^{\mathbb{R}}$, the $\boldsymbol{A}+\boldsymbol{B}$ Laplacian $C_{H ; A, B}:=-\Delta+\left.|A+B|_{H}\right|^{2}$ is $\mathcal{S}$-adapted.

(ii) The Jacobi field operator $J_{H}:=-\Delta_{H}-|A|^{2}-R i c_{M}(v, v)$ is shifted $\mathcal{S}$-adapted. If, in addition, $H \in \mathcal{H}$, then $J_{H}$ has a principal eigenvalue $\geq 0$.

These operators have two basic properties in common. First, they are naturally associated with any $H \in \mathcal{G}$. This means that there is a unique expression for $L(H)$ on $H \backslash \Sigma_{H}$ such that the assignment $H \mapsto L(H)$ commutes with the convergence of sequences of almost minimizers, cf. Ch. 3.2 for details. Second, they are Schrödinger operators with finite principal eigenvalues. We merge these properties into one concept (cf. also Chapters 4.1-4.2):

Definition 1.6 A natural and shifted $\mathcal{S}$-adapted operator $L$ is called a natural Schrödinger operator if for any given $H \in \mathcal{H}, L(H)$ has the form

$$
L(H)(u)=-\Delta_{H} u+V_{H}(x) \cdot u \text { on } H \backslash \Sigma_{H}
$$

for some Hölder continuous function $V_{H}(x)$.

Blow-Up Martin Theory We take the analysis of natural Schrödinger operators near singular points beyond the Martin theory on $H \backslash \Sigma_{H}$ by considering tangent cones and cone reduction arguments. These are common in the geometric study of (almost) minimizers, for instance to prove bounds on the codimension of their singularity sets. For natural Schrödinger operators we build a matching analytic reduction scheme. In view of the key role played by the boundary Harnack inequalities [17, Theorem 1 and 2] our goal is to 
understand how minimal growth towards singularities transfers to the associated induced solutions on the tangent cones obtained by blowing up.

The minimal growth concept we use in this context is that of solutions $L$-vanishing in (parts of) $\Sigma_{H}$. We recall from [17] that a solution $u>0$ of $L \phi=0$ in $p \in \widehat{\Sigma}$ is $\boldsymbol{L}$ vanishing in $p$ when there exists a supersolution $w>0$ with $u / w(x) \rightarrow 0$ for $x \rightarrow p$ with $x \in H \backslash \Sigma$.

The following Theorem asserts an inheritance of minimal growth properties under blowups of the underlying spaces. The remarkable point is that we are comparing the fine asymptotic analysis of distinct spaces with completely different singularity sets. We are not aware of any comparable result in the literature.

Theorem 3 (Minimal Growth and Blow-Ups, see Theorem 3.12) Let $H \in \mathcal{G}$ and $L$ be some natural Schrödinger operator on $H \backslash \Sigma$. Further, let $p \in \Sigma_{H}$ and $C$ be any tangent cone in $p$. If $L(H)$ is $\mathcal{S}$-adapted, then $L(C)$ is $\mathcal{S}$-adapted. Furthermore, if $u>0$ is any solution of $L(H) \phi=0$ which is $L$-vanishing in a neighborhood $V$ of $p$, then any solution of $L(C) \phi=0$ induced by $u$ is $L(C)$-vanishing along the entire singular set $\sigma_{C} \subset C$.

Remark 1.7 If we do not fix the singular point while we scale $H$, that is, we consider a converging subsequence of pointed spaces $\left(s_{i} \cdot H, p_{i}\right)$ for $p_{i} \rightarrow p, p_{i} \in \Sigma_{H}$, and $s_{i} \rightarrow \infty$ of scaling factors, then the limit space $\left(H_{\infty}, p_{\infty}\right)$ can be a general minimal hypersurface in $\mathcal{H}_{n}^{\mathbb{R}}$. In this case any induced solution on $H_{\infty} \backslash \Sigma_{H_{\infty}}$ is $L$-vanishing along $\Sigma_{H_{\infty}}$.

The $\mathcal{S}$-adaptedness of $L(C)$ implies in particular that Martin theory applies to $C \backslash \sigma_{C}$. By [17, Theorem 3] there is exactly one Martin boundary point $\Psi_{+}$at $\infty$. We emphasize that it is not the symmetry of the cone but the $\mathcal{S}$-uniformity of $C$ which implies uniqueness of $\Psi_{+}$. Indeed, Ancona gave striking counterexamples of Euclidean cones over non-uniform spherical domains with uncountable families of minimal Martin boundary points at infinity [1]. In turn, the uniqueness of $\Psi_{+}$combined with the cone symmetry of $C$ and the separation of variables for natural Schrödinger operators over cones yields the following structure result.

Theorem 4 (Separation of Variables, see Theorem 4.4) Let $C \in \mathcal{S C}_{n}$ and $L$ be a natural Schrödinger operator. For the $\mathcal{S}$-adapted operator $L_{\lambda}=L-\lambda \cdot\langle A\rangle^{2} \cdot I d, \lambda<\lambda_{L, C}^{\langle A\rangle}$, and the two distinguished points $\Psi_{-}$at zero and $\Psi_{+}$at infinity in the Martin boundary of $L_{\lambda}(C)$, we have, in terms of polar coordinates $(\omega, r)$ :

$$
\Psi_{ \pm}(\omega, r)=\psi(\omega) \cdot r^{\alpha_{ \pm}},(\omega, r) \in S_{C} \backslash \sigma \times \mathbb{R}^{>0}, \text { with } \alpha_{ \pm}=-\frac{n-2}{2} \pm \sqrt{\left(\frac{n-2}{2}\right)^{2}+\mu}
$$

for some constant $\mu(C, L, \lambda)>-(n-2)^{2} / 4$.

These results describe the behavior of the $\Psi_{ \pm}$on individual cones. In general, however, we have infinitely many distinct tangent cones around a singular point $p \in \Sigma_{H}$. Our next result is a variant of Theorem 3 asserting that the assignment of $\Psi_{ \pm}$to the underlying cones is natural in the following sense.

Theorem 5 (Naturality of $\Psi_{ \pm}$, see Theorem 3.13) Let $L$ be a natural and $\mathcal{S}$-adapted Schrödinger operator on cones $C \in \mathcal{S C}_{n}$. Then for any flat norm converging sequence $C_{i} \rightarrow C_{\infty}, i \rightarrow \infty$, with suitably normalized associated solutions $\Psi_{ \pm}\left(C_{i}\right)$ and $\Psi_{ \pm}\left(C_{\infty}\right)$, 
we have

$$
\Psi_{ \pm}\left(C_{i}\right) \circ \mathbf{I D} \rightarrow \Psi_{ \pm}\left(C_{\infty}\right) C^{2, \alpha} \text {-compactly on } C_{\infty} \backslash \sigma_{C_{\infty}} \text { as } i \rightarrow \infty,
$$

where ID is the asymptotic identification representing $C_{i}$ as a $C^{2, \alpha}$-section of the normal bundle over $C_{\infty}$, cf. Ch. 3.1 .

A more general version of this result applies to converging sequences in $\mathcal{H}_{n}^{\mathbb{R}}$, cf. 3.14.

With later applications in scalar curvature geometry and general relativity in mind, we derive more detailed results for the conformal Laplacians $L_{H}$ and $L_{C}$ on $H$ and its tangent cones $C$ :

Theorem 6 (Conformal Laplacians, see Theorem 4.5) There are constants $\Lambda_{n}>\lambda_{n}>0$ depending only on $n$ such that for $\lambda \in\left(0, \lambda_{n}\right]$ and any singular area minimizing cone $C,\left(L_{C}\right)_{\lambda}$ is $\mathcal{S}$-adapted. Furthermore, we have the following estimates for $\Psi_{ \pm}(\omega, r)=$ $\psi(\omega) \cdot r^{\alpha_{ \pm}}$:

- $0>\alpha_{+} \geq-\left(1-\sqrt{\frac{3}{4}}\right) \cdot \frac{n-2}{2}>-\frac{n-2}{2}>-\left(1+\sqrt{\frac{3}{4}}\right) \cdot \frac{n-2}{2} \geq \alpha_{-}>-(n-2)$

- $|\psi|_{L^{1}\left(S_{C} \backslash \Sigma_{S_{C}}\right)} \leq a_{n, \lambda} \cdot \inf _{\omega \in S_{C} \backslash \Sigma_{S_{C}}} \psi(\omega)$ for some constant $a_{n, \lambda}>0$ depending only on $n$ and $\lambda$.

An interesting point in this result is the uniform separation of the exponents given by the lower bound $\alpha_{+}-\alpha_{-} \geq \sqrt{3 / 4} \cdot(n-2)$.

Inductive Analysis Theorems 3-5 give us a recipe to carry out the asymptotic analysis of natural $\mathcal{S}$-adapted Schrödinger operators near $\Sigma \subset H$. Positive solutions on $H \backslash \Sigma$ of minimal growth induce minimal growth solutions on any of its (iterated) tangent cones along an entire tree of blow-ups. Then, we transfer insights for these solutions we get on these cones back to $H$. The result is the following top down to bottom up asymptotic analysis towards $p$ :

- Top Down We start with a solution on $H \backslash \Sigma_{H}$ of minimal growth near some $p \in \Sigma_{H}$, choose some tangent cone $C$ and consider the (uniquely determined) induced solution of minimal growth towards $\sigma_{C} \subset C$ on $C \backslash \sigma_{C}$. Now we blow-up in points of $\sigma_{C}$ distinct from the tip and iterate this process until we reach the elementary case of a product cone $\mathbb{R}^{m} \times C^{n-m}$, for some $C^{n-m} \subset \mathbb{R}^{n-m+1}$ singular only in 0 . This happens at the latest after $\operatorname{dim} H-7$ times. Since by uniqueness, the induced minimal solutions are $\mathbb{R}^{n-m+1}$-translation symmetric we end up with the explicit description provided by Theorem 4 over cones singular only at the tip. Theorem 5 takes care of the non-uniqueness of tangent cones and yields uniform control for all these cones.

- Bottom Up Then we collect and transfer pieces of information for the induced solutions on the various tangent cones backwards following this tree of blow-ups to the initially given $H$ from any such terminal node $\mathbb{R}^{m} \times C^{n-m}$, for some $C^{n-m} \subset \mathbb{R}^{n-m+1}$ singular only in 0 . This way we get an asymptotic portrait of the initially given solution on $H \backslash \Sigma$ near $p$ from the family of Martin theories on $H \backslash \Sigma$ and on its (iterated) tangent cones. 


\section{Hardy $\mathcal{S}$-Structures}

The goal of this chapter is to show that many classical operators are actually shifted $\mathcal{S}$ adapted with respect to a special subclass of $\mathcal{S}$-structures which we call Hardy $\mathcal{S}$-structures. Similar to Martin theory for $\mathcal{S}$-adapted operators, this is rather a property of the underlying space than of the operators.

\subsection{Hardy Inequalities}

The Poincaré inequality is a frequently used tool in geometric analysis. For a Lipschitz regular and bounded Euclidean domain $D \subset \mathbb{R}^{n}$ it asserts that there is some constant $a_{D}>0$ such that

$$
\int_{D}|\nabla \phi|^{2} d V \geq a_{D} \cdot \int_{D} \phi^{2} d V
$$

for any smooth function $\phi$ compactly supported in $D$. The Hardy inequality is a remarkable refinement of this result. Indeed, under the previous assumptions we can even find a constant $c_{D}>0$ such that for any such $\phi$,

$$
\int_{D}|\nabla \phi|^{2} d V \geq c_{D} \cdot \int_{D} \frac{\phi^{2}}{\operatorname{dist}(x, \partial D)^{2}} d V
$$

cf. the detailed expositions [2] and [13] for some background information.

Now for $H \in \mathcal{G}$, the singular set $\Sigma \subset H$ plays the role of a boundary of $H \backslash \Sigma$, and we shall prove similar Hardy inequalities as in the Euclidean case using the metric distance $\operatorname{dist}(x, \Sigma)$. We refer to these as metric Hardy inequalities. More importantly, we get for suitable $\mathcal{S}$-transforms a stronger $\mathcal{S}$-Hardy inequality using the $\mathcal{S}$-distance $\delta_{\langle A\rangle}(x)$. In fact, the metric Hardy inequalities are a simple byproduct of our $\mathcal{S}$-formalism, in the same way as uniformity of $H \backslash \Sigma$ follows from $\mathcal{S}$-uniformity [18].

We get two versions of Hardy inequalities according to whether $H$ is compact or lies in $\mathcal{H}_{n}^{\mathbb{R}}$. The latter case is the main case as it covers blow-up limits of hypersurfaces in $\mathcal{G}$ around singular points.

Theorem 2.1 (Hardy $\mathcal{S}$-Structures) There are $\mathcal{S}$-transforms $\langle A\rangle$ such that for all $H \in \mathcal{G}$ and $f \in C_{0}^{2}(H \backslash \Sigma)$, the space of $C^{2}$-functions compactly supported in $H \backslash \Sigma$, the following holds.

$\left(H_{c}\right) \quad$ For any compact $H \in \mathcal{G}^{c}$ and any $C^{\alpha}$-regular $(2,0)$-tensor $B$ on the ambient space $M$ of $H$ with $\left.B\right|_{H} \not \equiv-A_{H}$ there exists a constant $k_{H ; B}>0$ such that

$$
\int_{H}|\nabla f|^{2}+\left.|A+B|_{H}\right|^{2} \cdot f^{2} d V \geq k_{H ; B} \cdot \int_{H}\langle A\rangle^{2} \cdot f^{2} d V \geq \frac{k_{H ; B}}{L_{\langle A\rangle}^{2}} \cdot \int_{H} \frac{f^{2}}{\operatorname{dist}_{H}(x, \Sigma)^{2}} d V .
$$

For singular $H \in \mathcal{G}^{c},|A|$ is unbounded but $|B|_{H} \mid$ remains bounded, so that, in this case, the condition $\left.B\right|_{H} \not \equiv-A$ is redundant.

$\left(H_{\mathbb{R}}\right) \quad$ For $H \in \mathcal{H}_{n}^{\mathbb{R}}$ we only consider the case $B=0$. Then the Hardy constant depends solely on the dimension, that is, $k_{H ; 0}=k_{n}>0$, and we have

$$
\int_{H}|\nabla f|^{2}+|A|^{2} \cdot f^{2} d V \geq k_{n} \cdot \int_{H}\langle A\rangle^{2} \cdot f^{2} d V \geq \frac{k_{n}}{L_{\langle A\rangle}^{2}} \cdot \int_{H} \frac{f^{2}}{d i s t_{H}(x, \Sigma)^{2}} d V .
$$

These relations also apply to the case $\Sigma=\emptyset$ via the convention $1 / \operatorname{dist}(x, \Sigma)=0$. An $\mathcal{S}$-transform satisfying both axioms $(H)=\left(H_{c}\right)+\left(H_{\mathbb{R}}\right)$ is called a Hardy $\mathcal{S}$-transform. 
Remark 2.2 For compact $H \in \mathcal{G}$, we need both integrands $|\nabla f|^{2}$ and $\left.|A+B|_{H}\right|^{2}$ on the left hand side of these inequalities. Indeed, since $H$ is compact and $\operatorname{codim} \Sigma \geq 2$, the coarea formula (cf. for instance [11, Theorem 2.1.5.3]) shows that

$$
\inf _{f \in C_{0}^{2}(H \backslash \Sigma)} \int_{H}|\nabla f|^{2} d V / \int_{H}\langle A\rangle^{2} \cdot f^{2} d V=0 .
$$

On the other hand, $|A|$ usually vanishes or at least converges to zero along suitable sequences of points approaching $\Sigma$, while $\langle A\rangle$ converges to $+\infty$. (This corresponds to rays in the tangent cones along which the cones are totally geodesic). Then, for $C^{2}$-functions supported around such points, we get

$$
\left.\inf _{f \in C_{0}^{2}(H \backslash \Sigma)} \int_{H}|A+B|_{H}\right|^{2} \cdot f^{2} d V / \int_{H}\langle A\rangle^{2} \cdot f^{2} d V=0 .
$$

Since the Hardy inequalities $\left(H_{c}\right)$ and $\left(H_{\mathbb{R}}\right)$ do not follow from the $\mathcal{S}$-axioms we have to revisit our construction of metric $\mathcal{S}$-transforms $\langle A\rangle_{\zeta, H}$ which we briefly recall. If $H \in \mathcal{G}$ is totally geodesic, we set $\langle A\rangle_{\zeta, H} \equiv 0$. Otherwise, for $c>0$ we set $\mathbb{A}_{c}(\varsigma):=$ the boundary of the $\varsigma / c$-distance tube $\mathbb{U}_{c}^{\varsigma}$ of $|A|^{-1}[c, \infty)$ and define

$$
\langle A\rangle_{\varsigma, H}(x):=\sup \left\{c \mid x \in \overline{\mathbb{U}}_{c}^{\varsigma}\right\}, \quad x \in H \backslash \Sigma .
$$

A key input in proving that $\langle A\rangle_{\varsigma}$ also satisfies $(H)=\left(H_{c}\right)+\left(H_{\mathbb{R}}\right)$ will be a quantitative version of the fact that tangent cones in singular points are also singular, cf. [18, A.II.] for some background.

Proposition 2.3 For any $\varsigma>0$ the metric $\mathcal{S}$-transform $\langle A\rangle_{\varsigma}$ is a Hardy $\mathcal{S}$-transform.

Remark 2.4 Apart of the proof of Proposition 2.3 our later applications shall only make use of the axioms (S1)-(S4) and (H), but not of any particular feature of $\langle A\rangle_{\varsigma}$. Once we have proven this result we therefore simply add $(\mathrm{H})$ to our set of $\mathcal{S}$-axioms and henceforth assume that all our $\mathcal{S}$-transforms are Hardy $\mathcal{S}$-transforms.

For the proof of Proposition 2.3 we may assume that $H$ is not totally geodesic, whence $\langle A\rangle_{\varsigma}>0$. Otherweise $H$ is totally geodesic, so $\langle A\rangle_{\varsigma}(x) \equiv 0$, and the Hardy inequalities hold trivially. We also note that the largest constant $k=k_{\zeta, H}$ such that

$$
\int_{H}|\nabla f|^{2}+\left.|A+B|_{H}\right|^{2} \cdot f^{2} d V \geq k \cdot \int_{H}\langle A\rangle_{\varsigma}^{2} \cdot f^{2} d V
$$

holds for any $f \in C_{0}^{2}(H \backslash \Sigma)$ is nothing but the first eigenvalue $\lambda_{P_{\varsigma}}$ of the weighted operator

$$
P_{\varsigma} u:=\langle A\rangle_{\varsigma}^{-2} \cdot\left(-\Delta u+\left.|A+B|_{H}\right|^{2} \cdot u\right) .
$$

Since $\langle A\rangle_{\varsigma}$ is locally Lipschitz, elliptic theory shows that eigenfunctions of $P_{\varsigma}$ are $C^{2, \alpha}{ }_{-}$ regular, cf. [12, Chapter 6.4]. Then $\lambda_{P_{\varsigma}}$ can be written as a Rayleigh quotient

$$
\lambda_{P_{\varsigma}}=\inf _{f \in C_{0}^{2}(H \backslash \Sigma), f \neq 0} \int_{H}|\nabla f|^{2}+\left.|A+B|_{H}\right|^{2} \cdot f^{2} d V / \int_{H}\langle A\rangle_{\varsigma}^{2} \cdot f^{2} d V .
$$

To estimate $\lambda_{P_{S}}$ we localize the problem to Neumann eigenvalues on regular balls. Then we take covers by such balls with controlled covering numbers and use them to derive a positive lower estimate for the eigenvalue $\lambda_{P_{5}}$. 


\subsection{Neumann Eigenvalues on Balls}

The Neumann eigenvalues of $P_{\varsigma}$ on balls are scaling invariant: For any ball $B_{r}(p) \subset H \backslash \Sigma$, $r>0$ and scaling factor $\mu>0$ we have

$$
v_{\varsigma}\left(B_{r}(p)\right)=v_{\varsigma}\left(\mu \cdot B_{r}(p)\right),
$$

for the integrands of the Rayleigh quotient (2) $|\nabla f|^{2}+\left.|A+B|_{H}\right|^{2}$ (numerator) and $\langle A\rangle^{2}$ (denominator) rescale by the same factor $\mu^{-2}>0$.

Next we would like to establish a lower bound for $v_{\zeta}\left(B_{r}(p)\right)$. However, there is no uniform positive lower bound for $r \rightarrow 0$ if $|A|(p)=0$. Conversely, when the balls become too large, we can neither control their geometry nor understand the eigenvalues or the covering numbers. On the other hand, when we approach $\Sigma$ we get better and better local approximations by Euclidean hypersurfaces in $\mathcal{H}_{n}^{\mathbb{R}}$ (after rescaling to a unit size). They are singular and real analytic hypersurfaces in $\mathbb{R}^{n+1}$. This leads us to the idea to let $\langle A\rangle_{\varsigma}$ determine the radius of the balls when we are close to $\Sigma$. Towards this end we notice that $\langle A\rangle_{\zeta}(x) \geq \varsigma /$ dist $_{H}(x, \Sigma)$ means that $B_{\zeta /\langle A\rangle_{\zeta}(p)}(p) \cap \Sigma=\emptyset$ for any $p \in H \backslash \Sigma$. For these balls we have the following estimates:

Lemma 2.5 For any $\varsigma \in(0,1]$ and $\mu \in(0,1 / 2)$ there is a neighborhood $U_{\zeta, \mu}$ of $\Sigma$ as well as a constant $\zeta\left(U_{\zeta, \mu}\right)>0$ such that

$$
v_{\zeta}\left(B_{\mu \cdot \zeta /\langle A\rangle_{\zeta}(p)}(p)\right) \geq \zeta \text { for any } p \in U_{\zeta, \mu} \backslash \Sigma \text {. }
$$

Proof To simplify notation we only consider the case $\varsigma=1$. We will make explicit use of the definition of $\langle A\rangle_{1}$. Let us assume that there is a sequence of points $p_{i} \in H \backslash \Sigma$ with $p_{i} \rightarrow p_{\infty} \in \Sigma$ and such that $\nu_{1}\left(B_{\mu /\langle A\rangle\left(p_{i}\right)}\left(p_{i}\right)\right) \rightarrow 0$. After scaling $B_{\mu /\langle A\rangle_{1}\left(p_{i}\right)}$ by $\langle A\rangle_{1}\left(p_{i}\right)$ to obtain $B_{\mu}\left(p_{i}\right) \subset\langle A\rangle_{1}\left(p_{i}\right) \cdot H$ we may assume that

$$
\sup \left\{|A|(x) \mid x \in B_{1}\left(p_{i}\right) \cap\langle A\rangle_{1}\left(p_{i}\right) \cdot H\right\}=1
$$

and

$$
\sup \left\{|B|(x) \mid x \in B_{1}\left(p_{i}\right) \cap\langle A\rangle_{1}\left(p_{i}\right) \cdot H\right\} \leq 1 / i
$$

in view of the definition of $\langle A\rangle$, the boundedness of $|B|$ and $|B|_{\lambda M}=\lambda^{-1} \cdot|B|_{M}, \lambda>0$. Moreover, taking without loss of generality $p_{\infty}=0$, we get a subsequence of the pointed spaces $\left(\langle A\rangle_{1}\left(p_{i}\right) \cdot H, p_{i}\right)$ which converge compactly in flat norm and thus in $C^{2, \alpha}$-norm to $\left(H_{\infty}, 0\right) \subset\left(\mathbb{R}^{n+1}, 0\right)$ by standard regularity theory. We notice that

$$
\sup \left\{|A|(x) \mid x \in B_{1}\left(p_{i}\right) \cap\langle A\rangle_{1}\left(p_{i}\right) \cdot H\right\}=1
$$

and thus $\langle A\rangle_{1}(0)=1$. Here we use the following inequality [18, Lemma A.8]: For any $\mu \in(0,1]$, there is a constant $c(\mu, n)>0$ such that

$$
\sup \left\{|A|(x) \mid x \in B_{\mu}(0) \cap H_{\infty}\right\} \geq c .
$$

We claim that the first Neumann eigenvalue $v_{1}\left(B_{\mu}(0) \cap H_{\infty}\right)$ for $P_{1}$ on $B_{\mu}(0) \cap H_{\infty}$ is positive:

$$
\inf _{f \in C^{2}\left(B_{\mu}(0) \cap H_{\infty}\right), f \not \equiv 0} \int_{B_{\mu}(0) \cap H_{\infty}}|\nabla f|^{2}+|A|^{2} \cdot f^{2} d V / \int_{B_{\mu}(0) \cap H_{\infty}}\langle A\rangle_{1}^{2} \cdot f^{2} d V>0 .
$$

Note that the tensor $B$ vanishes in the limit since as $\lambda \rightarrow \infty$ for $\lambda^{-1} \cdot|B|_{M} \rightarrow 0$ in $C^{2, \alpha}$ norm. Since there is a positive upper bound for $\langle A\rangle_{1}$ over $B_{\mu}(0) \cap H_{\infty}$, it suffices to consider 
the usual non-weighted Neumann eigenvalue

$$
v\left(B_{\mu}(0) \cap H_{\infty}\right)=\inf _{f \in C^{2}\left(B_{\mu}(0) \cap H_{\infty}\right), f \neq 0} \int_{B_{\mu}(0) \cap H_{\infty}}|\nabla f|^{2}+|A|^{2} \cdot f^{2} d V / \int_{B_{\mu}(0) \cap H_{\infty}} f^{2} d V .
$$

Clearly, $v\left(B_{\mu}(0) \cap H_{\infty}\right) \geq 0$, and for $v\left(B_{\mu}(0) \cap H_{\infty}\right)=0$, we would have a positive $C^{2}$ function $u$ with $\Delta u=|A|^{2} \cdot u$ and vanishing normal derivative along $\partial B_{\mu}(0) \cap H_{\infty}$. But then Stokes' theorem would imply $\int_{B_{\mu}(0) \cap H_{\infty}} \Delta u=0$, whereas $\int_{B_{\mu}(0) \cap H_{\infty}}|A|^{2} \cdot u>0$. Hence for sufficiently large $i$,

$$
v_{1}\left(B_{\mu /\langle A\rangle_{1}\left(p_{i}\right)}\left(p_{i}\right)\right) \geq v_{1}\left(B_{\mu}(0) \cap H_{\infty}\right) / 2>0
$$

which contradicts $v_{1}\left(B_{\mu /\langle A\rangle_{1}\left(p_{i}\right)}(p)\right) \rightarrow 0$.

\subsection{Controlled Covers}

To derive Proposition 2.3, we combine these estimates for Neumann eigenvalues with the following $\mathcal{S}$-adapted covers for $H \in \mathcal{H}$ introduced in [18, Proposition B.1] to construct the $\mathcal{S}$-Whitney smoothings (which equally apply to $H \in \mathcal{G}$ since the whole argument is based on blow-up arguments to limits in $\mathcal{H}^{\mathbb{R}}$ ). For a consistent statement we also include the trivial case of totally geodesic $H \in \mathcal{G}$.

Proposition 2.6 (S-Adapted Covers) There exists some $\xi_{0}\left(n, L_{\langle A\rangle_{\varsigma}}\right) \in\left(0,1 /\left(10^{3} \cdot L_{\langle A\rangle_{\varsigma}}\right)\right)$ with the following property. For every $H \in \mathcal{G}$ and for every $\xi \in\left(0, \xi_{0}\right)$ there is a locally finite covering $\mathcal{A}=\left\{\overline{B_{\Theta(p)}} \mid p \in A\right\}$ of $H \backslash \Sigma$ by closed balls of radius $\Theta(p):=$ $\xi /\langle A\rangle_{\zeta}(p)=\xi \cdot \delta_{\langle A\rangle_{\varsigma}}(p)$ and some discrete set $A \subset H \backslash \Sigma$, such that for a suitably small neighborhood $Q$ of $\Sigma$ we have:

(C1) For $p \in Q$ the exponential map $\left.\exp _{p}\right|_{B_{100 \Theta(p)}(0)}$ is bi-Lipschitz onto its image for some bi-Lipschitz constant $l(n) \geq 1$.

(C2) $\quad A^{Q}:=A \cap Q$ splits into $c(n)$ disjoint families $A^{Q}(1), \ldots, A^{Q}(c)$ with

(i) $B_{10 \Theta(p)}(p) \cap B_{10 \Theta(q)}(q)=\emptyset$, for $p$ and $q$ in the same $A^{Q}(k)$,

(ii) $q \notin \overline{B_{\Theta(p)}(p)}$, for any two $p, q \in A^{Q}$.

In particular, for any $z \in Q$ and $\rho \in(0,10)$ the covering number

$$
\#(A \cap Q, z, \rho):=\#\left\{x \in A \cap Q \mid z \in B_{\rho \cdot \Theta(x)}(x)\right\} \leq c(n)
$$

by balls centered in $A \cap Q$ is uniformly bounded. Such a cover $\mathcal{A}$ will be called $\mathcal{S}$-adapted. These covers have the following properties.

(i) For $H \in \mathcal{H}^{\mathbb{R}}$ we may choose $Q=H \backslash \Sigma$.

(ii) For any $\varepsilon>0$, there is $a \xi_{\varepsilon} \in\left(0, \xi_{0}\left(n, L_{\langle A\rangle}\right)\right)$ such that for every $p \in H \backslash \Sigma$ the exponential map $\left.\exp _{p}\right|_{B_{100 \cdot \xi_{\varepsilon} /\langle A\rangle_{\varsigma}(p)}(0)}$ is bi-Lipschitz onto its image with bi-Lipschitz constant $1+\varepsilon$.

Proof of Proposition 2.3 We first consider $\left(H_{c}\right)$. We start with the simplest case where $H$ is totally geodesic. Then $\langle A\rangle_{\varsigma} \equiv 0$ and the Hardy inequality becomes trivial. Thus we take $H$ not totally geodesic so that $\langle A\rangle_{\varsigma}>0$ on $H$.

Assuming that $H$ is regular we have an upper bound for $\langle A\rangle_{\varsigma}>0$ on $H$. It is therefore enough prove the positivity of the usual eigenvalue of $-\Delta+\left.|A+B|_{H}\right|^{2}$. Now 
$\left.|A+B|_{H}\right|^{2} \geq 0$, and in some open set it is positive since $\left.B\right|_{H} \not \equiv-A$. Thus, for any positive $C^{2}$-function $u$ (including the first eigenfunction) we have $\int_{H} \Delta u=0$, whereas $\left.\int_{H}|A+B|_{H}\right|^{2} \cdot u>0$. Hence, the eigenvalue of $-\Delta+\left.|A+B|_{H}\right|^{2}$ cannot be zero.

Now we turn to the main case where $H$ is singular. As above we notice that $v_{\varsigma}(H \backslash \bar{W})>0$ for sufficiently small and smoothly bounded neighborhoods $W$ of $\Sigma \subset H$. Indeed, $H \backslash \bar{W}$ contains a non-empty open ball where $|A+B|_{H} \mid>0$ if $W$ is sufficiently small, since there are $p_{k} \in H \backslash \Sigma$ where $|A|\left(p_{k}\right) \geq k$, for any $k \geq 1$, whereas $|B|_{H} \mid$ remains bounded. For fixed $W$ we have positive bounds for $\langle A\rangle_{\varsigma}$, and we can consider the standard Neumann eigenfunction. Again we can invoke Stokes' Theorem to infer that $v_{\zeta}(H \backslash \bar{W})>0$. We note in passing that once we found a neighborhood $W$ with $v_{\varsigma}(H \backslash \bar{W})>0$, positivity continues to hold for all neighborhoods $W^{*}$ of $\Sigma$ with $W^{*} \subset W$. However, this argument does not give a uniform positive lower bound while $W^{*}$ shrinks to $\Sigma$ since $\langle A\rangle_{\varsigma}$ diverges when we approach $\Sigma$.

Next we take an $\mathcal{S}$-adapted cover for $\xi=\mu \cdot \varsigma$ and radii $\Theta(p)=\mu \cdot \varsigma /\langle A\rangle_{\varsigma}(p)$, and we choose $W \subset Q$, so that $\mathcal{A}_{Q}=\left\{\overline{B_{\Theta(p)}} \mid p \in Q\right\}$ is covering of $W$ with covering number $c(n)$. To ease notation we will write $B(p)=B_{\mu \cdot \varsigma /\langle A\rangle_{\varsigma}(p)}(p)$. Then for any $f \in$ $C_{0}^{\infty}(H \backslash \Sigma), f \not \equiv 0$ we get the following estimate:

$$
\begin{aligned}
& \int_{H}|\nabla f|^{2}+\left.|A+B|_{H}\right|^{2} \cdot f^{2} d V \\
\geq & \frac{1}{c(n)+1} \cdot\left(\int_{H \backslash \bar{W}}|\nabla f|^{2}+\left.|A+B|_{H}\right|^{2} \cdot f^{2} d V+\sum_{B(p) \in \mathcal{A}} \int_{B(p)}|\nabla f|^{2}+\left.|A+B|_{H}\right|^{2} \cdot f^{2} d V\right) \\
\geq & \frac{1}{c(n)+1} \cdot\left(v_{\zeta}(H \backslash \bar{W}) \cdot \int_{H \backslash \bar{W}}\langle A\rangle_{\zeta}^{2} \cdot|f|^{2} d V+\sum_{B(p) \in \mathcal{A}} v_{\zeta}(B(p)) \cdot \int_{B(p)}\langle A\rangle_{\zeta}^{2} \cdot|f|^{2} d V\right) \\
\geq & \frac{1}{c(n)+1} \cdot \min \left\{v_{S}(H \backslash \bar{W}), \inf _{B(p) \in \mathcal{A}} v_{S}(B(p))\right\} \cdot \int_{H}\langle A\rangle_{\zeta}^{2} \cdot|f|^{2} d V .
\end{aligned}
$$

On the other hand, the Neumann eigenvalues $v_{\zeta}(B(p))$ are uniformly bounded from below by $v_{\varsigma}\left(B_{\mu \cdot \varsigma /\langle A\rangle_{\varsigma}(p)}(p)\right) \geq \zeta$, cf. (4) in Lemma 2.5 , whence

$$
\lambda_{P_{\zeta}} \geq \frac{\min \left\{v_{\zeta}(H \backslash \bar{W}), \zeta\right\}}{c(n)+1}>0 .
$$

Put differently the Hardy inequality holds for $k_{\zeta, H}:=\lambda_{P_{\zeta}}$.

These arguments apply equally well in the case $H \in \mathcal{H}^{\mathbb{R}}$. In this case Proposition 2.6 asserts that we get $\mathcal{S}$-adapted covers not only of small neighborhoods of $\Sigma$, but of the entire hypersurface. Hence the previous chain of inequalities now yields

$$
\begin{aligned}
& \int_{H}|\nabla f|^{2}+|A|^{2} \cdot f^{2} d V \\
\geq & \frac{1}{c(n)} \cdot \sum_{B(p) \in \mathcal{A}} \int_{B(p)}|\nabla f|^{2}+|A|^{2} \cdot f^{2} d V \\
\geq & \frac{1}{c(n)} \cdot \sum_{B(p) \in \mathcal{A}} v_{\varsigma}(B(p)) \cdot \int_{B(p)}\langle A\rangle_{\varsigma}^{2} \cdot|f|^{2} d V .
\end{aligned}
$$


Therefore, the Hardy inequality holds for any $H \in \mathcal{H}^{\mathbb{R}}$ and for $k_{\zeta, n}:=\zeta / c(n)$.

\subsection{Geometric Operators}

For the remainder of this paper we fix a Hardy $\mathcal{S}$-transform $\langle A\rangle$ on $\mathcal{G}$. In this section we shall employ the Hardy axiom $(H)$ to verify the $\mathcal{S}$-adaptedness of some basic geometric operators.

The natural geometric operator

$$
C_{H ; A, B}:=-\Delta+\left.|A+B|_{H}\right|^{2},
$$

which we call the $\mathbf{A}+\mathbf{B}$ Laplacian, couples to the second fundamental form of $H \subset M$ and an additional $(2,0)$-tensor $B$ on $M$. Of course, this definition is suggested right from the definition of Hardy $\mathcal{S}$-transforms, and the operator will be useful to provide lower bounds for variational integrals involving other $\mathcal{S}$-adapted operators.

Lemma 2.7 (A+B Laplacian) For any $C^{\alpha}$-regular (2,0)-tensor $B$ on $M$ with $\left.B\right|_{H} \not \equiv-A$ if $H \in \mathcal{G}_{n}^{c}$ and with $B=0$ if $H \in \mathcal{H}_{n}^{\mathbb{R}}$, the $A+B$ Laplacian $C_{H ; A, B}$ is an $\mathcal{S}$-adapted operator.

Proof Theorem 2.1 shows the $\langle A\rangle$-finiteness of $C_{H ; A, B}$ for a positive $\tau>0$. Thus we only need to verify the $\langle A\rangle$-adaptedness. With respect to the charts $\psi_{p}$ we write

$$
-\Delta u+\left.|A+B|_{H}\right|^{2} \cdot u=\sum_{i, j} a_{i j} \cdot \frac{\partial^{2} u}{\partial x_{i} \partial x_{j}}+\sum_{i} b_{i} \cdot \frac{\partial u}{\partial x_{i}}+c \cdot u .
$$

We recall that in local coordinates the Laplacian $\Delta u$ equals $\frac{1}{\sqrt{\text { det } g}} \cdot \sum_{i, j} \frac{\partial}{\partial x_{i}}\left(\sqrt{\text { det } g} \cdot g^{i j}\right.$. $\left.\frac{\partial u}{\partial x_{j}}\right)$ and that $\left.|A+B|_{H}\right|^{2}$ is $C^{\alpha}$. Hence the coefficients $a_{i j}$ are $C^{1}$ and $b_{i}, c$ are $C^{\alpha}$. Since the charts $\psi_{p}$ are the geodesic coordinates around $p$ we have with respect to these charts $a_{i j}(p)=\delta_{i j}, b_{i}(p)=0$ and $c(p)=\left.|A+B|_{H}\right|^{2}(p)$. Moreover, $|B|_{H} \mid$ remains bounded. Thus, the unfolding correspondence [17, Proposition 3.3] shows that $\delta_{\langle A\rangle^{*}}^{2} \cdot L$ is an adapted operator on $\left(H \backslash \Sigma, d_{\langle A\rangle^{*}}\right)$.

One source for $\mathcal{S}$-adapted operators are geometric and physical problems. Here, geometric properties of the ambient space like its curvature, or a given tensor $T$ coming from physical constraints, can translate into shifted $\mathcal{S}$-adapted operators on the hypersurface. We usually get better estimates in the case of area minimizers $H \in \mathcal{H}$ than is the general case of almost minimizers $H \in \mathcal{G}$, where we use boldface symbols for easier identification.

Theorem 2.8 (Curvature Constraints) Let $\langle A\rangle$ be a Hardy $\mathcal{S}$-transform. Further, let $H=$ $H^{n} \in \mathcal{G}_{n}$ with $H^{n} \subset M^{n+1}$ and such that $H \backslash \Sigma$ is non-totally geodesic. Then we have on $H \backslash \Sigma$ :

(i) The conformal Laplacian $L_{H}:=-\Delta_{H}+\frac{n-2}{4(n-1)} \cdot$ scal $_{H}$ is shifted $\mathcal{S}$-adapted. If, in addition, scal ${ }_{M} \geq 0$ and $H \in \mathcal{H}$, then $L_{H}$ is even $\mathcal{S}$-adapted.

(ii) More generally, let $S$ be any bounded $C^{\alpha}$-function on $M$. Then the $S$-conformal Laplacian $L_{H, S}:=-\Delta_{H}+\frac{n-2}{4(n-1)} \cdot\left(\right.$ scal $\left._{H}-\left.S\right|_{H}\right)$ is shifted $\mathcal{S}$-adapted. If, in addition, scal $_{M} \geq S$ and $H \in \mathcal{H}$, then $L_{H, S}$ is even $\mathcal{S}$-adapted. 
(iii) The Laplacian $-\Delta_{H}$ is shifted $\mathcal{S}$-adapted. For general $H \in \mathcal{G}$ the principal eigenvalue is non-negative $\lambda_{-\Delta, H}^{\langle A\rangle} \geq 0$. For $H \in \mathcal{G}^{c}$ the principal eigenvalue $\lambda_{-\Delta, H}^{\langle A\rangle}$ vanishes and the ground state is given by a constant function and $H \backslash \Sigma$ has the Liouville property saying that all bounded harmonic functions are constant.

(iv) For any $C^{\alpha}$-regular (2,0)-tensor $B$ on the ambient space $M$, with $\left.B\right|_{H} \not \equiv-A$ if $H \in$ $\mathcal{G}^{c}$ and with $B=0$ if $H \in \mathcal{H}_{n}^{\mathbb{R}}$, the $\boldsymbol{A}+\boldsymbol{B}$ Laplacian $C_{H ; A, B}:=-\Delta+\left.|A+B|_{H}\right|^{2}$ is $\mathcal{S}$-adapted.

(v) The Jacobi field operator $J_{H}:=-\Delta_{H}-|A|^{2}-R i c_{M}(v, v)$ is shifted $\mathcal{S}$-adapted. If, in addition, $H \in \mathcal{H}$, then $J_{H}$ has a principal eigenvalue $\geq 0$.

Proof By the arguments of Lemma 2.7, the operators are adapted to $\langle A\rangle$. To show the shifted $\mathcal{S}$-adaptedness for a general $H \in \mathcal{G}$ we first recall the Gauß-Codazzi equation

$$
\left|A_{H}\right|^{2}+2 \operatorname{Ric}_{M}(v, v)=\operatorname{scal}_{M}-\operatorname{scal}_{H}+\left(\operatorname{tr} A_{H}\right)^{2},
$$

where $\operatorname{tr} A_{H}$ is the mean curvature of $H$. When we approach $\Sigma$ on $H \backslash \Sigma$ we have:

(i) $\operatorname{Ric}_{M}(v, v) . \operatorname{scal}_{M}$ and, by assumption, $S$ remain bounded, whereas $\left|A_{H}\right|^{2}$ and $\operatorname{scal}_{H}$ diverge.

(ii) For $\operatorname{tr} A_{H}$ we have $\left(\operatorname{tr} A_{H}\right)^{2} \leq n \cdot\left|A_{H}\right|^{2}$. When $H$ is minimal we have $\operatorname{tr} A_{H}=0$ and if in addition $H \in \mathcal{H}_{n}^{\mathbb{R}}$ then Eq. 6 reduces to scal $_{H}=-\left|A_{H}\right|^{2}$.

(iii) For $H \in \mathcal{G}^{c}$ we also have $\langle A\rangle \geq c$, for some $c(H)>0$.

This shows the shifted $\mathcal{S}$-adaptedness of $L_{H}$ for any $H \in \mathcal{G}$ : using $\langle A\rangle \geq|A|$ and Eq. 6 there is some $k \geq 0$ so that for any $C^{2}$-function $f$ with supp $f \subset H \backslash \Sigma$ :

$\int_{H}|\nabla f|^{2}+\frac{n-2}{4(n-1)} \cdot \operatorname{scal}_{H} \cdot f^{2} d V \geq \int_{H}|\nabla f|^{2}-k \cdot\left(|A|^{2}+1\right) \cdot f^{2} d V \geq-(k+k / c) \cdot \int_{H}\langle A\rangle^{2} \cdot f^{2} d V$

The shifted $\mathcal{S}$-adaptedness of the other operators in the Theorem follows similarly.

We now turn to $L_{H, S}$ on an area minimizing hypersurface $H$. In this case, the second variation of the area functional $\mathbf{A}$ is non-negative. Hence, if $f$ is a $C^{2}$ function on $H$ with supp $f \subset H \backslash \Sigma$ and $v$ the outward normal vector field over $H \backslash \Sigma$, we get:

$\mathbf{A}^{\prime}(f)=\int_{H} \operatorname{tr} A_{H}(z) \cdot f(z) d V=0$ and $\mathbf{A}^{\prime \prime}(f)=\int_{H}|\nabla f|^{2}-\left(|A|^{2}+R i c_{M}(v, v)\right) \cdot f^{2} d V \geq 0$.

The Gauß-Codazzi Eq. 6 gives an equivalent formulation of $\mathbf{A}^{\prime \prime}(f) \geq 0$, namely

$$
\begin{aligned}
& \int_{H} f \cdot L_{H} f d V=\int_{H}|\nabla f|^{2}+\frac{n-2}{4(n-1)} \cdot \operatorname{scal}_{H} \cdot f^{2} d V \\
\geq & \int_{H} \frac{n}{2(n-1)} \cdot|\nabla f|^{2}+\frac{n-2}{4(n-1)} \cdot\left(|A|^{2}+\operatorname{scal}_{M}\right) \cdot f^{2} d V .
\end{aligned}
$$

Further, assuming $\operatorname{scal}_{M} \geq S$ gives

$$
\begin{aligned}
& \int_{H} f \cdot L_{H, S} f d V=\int_{H}|\nabla f|^{2}+\frac{n-2}{4(n-1)} \cdot\left(\operatorname{scal}_{H}-\left.S\right|_{H}\right) \cdot f^{2} d V \\
\geq & \int_{H} \frac{n}{2(n-1)} \cdot|\nabla f|^{2}+\frac{n-2}{4(n-1)} \cdot\left(|A|^{2}+s c a l_{M}-\left.S\right|_{H}\right) \cdot f^{2} d V \\
\geq & \frac{n-2}{4(n-1)} \cdot \int_{H} f \cdot C_{H ; A, 0} f d V \\
\geq & \tau(\langle A\rangle, H) \cdot \frac{n-2}{4(n-1)} \cdot \int_{H}\langle A\rangle^{2} \cdot f^{2} d V, \text { for } \tau(\langle A\rangle, H)>0 .
\end{aligned}
$$


For the Laplacian on $H \in \mathcal{G}$, the constant function $\equiv 1$ is a solution and thus the criticality theory [17, Ch.5.3] shows that $\lambda_{-\Delta, H}^{\langle A\rangle} \geq 0$. For $H \in \mathcal{G}^{c}$ we have $\lambda_{-\Delta, H}^{\langle A\rangle}=0$ as can be seen from choosing a cut-off of a positive constant function towards $\Sigma$. The coarea formula, cf. [11, Theorem 2.1.5.3], and the fact that the codimension of $\Sigma$ is $\geq 2$ show that there is no positive lower bound for $\int|\nabla f|^{2} d V$, with $\int f^{2} d V=1$. Moreover, $v:=u-\inf _{H \backslash \Sigma} u+1$ is a positive harmonic function for any bounded harmonic function $u$. Hence $v$, and therefore $u$, are constant functions.

Finally, we consider the Jacobi field operator $J_{H}$. For $H \in \mathcal{H}$ we get from Eq. 7:

$$
\int_{H} f \cdot J_{H} f d V=\int_{H}\left|\nabla_{H} f\right|^{2}-\left(|A|^{2}+\operatorname{Ric}_{M}(v, v)\right) \cdot f^{2} d V \geq 0
$$

for any $C^{2}$ function $f$ on $H$ with supp $f \subset H \backslash \Sigma$.

\section{Blow-Up Martin Theory}

The structure of the singular set $\Sigma_{H}$ of a given $H \in \mathcal{G}$ may be rather different from the structure of $\sigma_{C}$ for any of its tangent cones $C$. Nevertheless, for $\mathcal{S}$-adapted operators which are naturally associated with minimizers, we can derive non-trivial relations between the Martin theory on $H$ and that on its tangent cones. This is essential to extend tangent cone reduction techniques, classically applied to the geometry of $H$ and $\Sigma$, to the elliptic analysis on $H \backslash \Sigma_{H}$ near $\Sigma_{H}$.

\subsection{Almost Minimizers and ID-Maps}

Our understanding of almost minimizers entails a local graphical presentation of $H \in \mathcal{G}$ near singular points over its tangent cones. In this section we want to properly understand these graphical maps refining our discussions in [17] and [18, Appendix A]. To motivate our approach we briefly review some of the ideas originally developed to treat the model case of area minimizers.

Area Minimizing Boundaries The partial regularity theory for area minimizing oriented Euclidean boundaries $H \subset \mathbb{R}^{n+1}$, pioneered by De Giorgi, shows that $H \backslash \Sigma$ is $C^{\infty}$, in fact, analytic cf. [10, Ch.8]. The argument has two main steps. The deep step 1 shows that $H \backslash \Sigma$ makes up most of $H$ and that $H \backslash \Sigma$ is $C^{1}$-regular. Step 2 upgrades the regularity of the locally $C^{1}$-graphical representation of $H$ to $C^{\infty}$. This is elliptic regularity theory applied to the equation describing the vanishing mean curvature condition for the minimal surface $H$.

Turning to tangent cones we fix any basepoint $p \in \Sigma \subset H \subset \mathbb{R}^{n+1}$. We may assume $p=0 \in \mathbb{R}^{n+1}$. For any sequence $\tau_{i} \rightarrow \infty$, the blow-up sequence $H_{i}:=\tau_{i} \cdot H$ around $p$ converges in flat norm to an area minimizing tangent cone $C^{n} \subset \mathbb{R}^{n+1}$ after selecting some subsequence [10,9.3]. Now we choose some $q \in C$ with $\overline{B_{R}(q)} \subset C \backslash \sigma_{C}$, for some $R>0$. Allard regularity theory [20, 23.1] and elliptic theory, as in step 2 above, show:

Proposition 3.1 (ID-maps) For any $k \in \mathbb{Z}^{\geq 1}$ and large $i, B_{R}(q i) \cap H_{i}$, for some $q_{i} \in H \backslash \Sigma$ is a local $C^{k}$-section, up to minor adjustments near $\partial B_{R}(q)$

$$
\Gamma_{i}: B_{R}(q) \cap C \rightarrow B_{R}\left(q_{i}\right) \cap H_{i} \subset v \text { of the normal bundle } v \text { of } B_{R}(q) \cap C
$$


and, for $i \rightarrow \infty, \Gamma_{i}$ converges, in $C^{k}$-norm, to the zero section, which we identify with $B_{R}(q) \cap H_{i}$. We call the $C^{k}$-section ID $:=\Gamma_{i}$ the asymptotic identification map or ID-map for short. ${ }^{1}$

The Allard regularity argument and the definition of ID-maps equally applies to arbitrary flat norm converging sequences of area minimizers $H_{i}$ with some limit area minimizer $H_{\infty}$.

The point about the ID-map notion is that $C^{k}$-functions on $B_{R}\left(q_{i}\right) \cap H_{i}$ can be compared on common grounds to $C^{k}$-functions on $B_{R}(q) \cap C$ from an ID-map pull-back to $C$ (or $H_{\infty}$ ).

We also note that this regularity theory merely uses local data, that is, everything admits straightforward generalizations, using local charts, to the case of oriented boundaries $H \subset M^{n+1}$, when $M$ is a smooth Riemannian manifold. To study global aspects of area minimizers in $M^{n+1}$, one usually considers the more general mass minimizing currents cf. [18, A.IV] for some basics. For the present we only note that, using the strict maximum principle for area minimizers, these currents can locally be written as a sum (with multiplicity) of locally disjoint oriented boundaries, cf.[11, Theorem 7 in 4.3.1], [18, A.11]. That is, mass minimizing currents and their local graphical presentation over tangent cones can largely be understood from oriented boundaries.

Almost Minimizers The regularity theory for area minimizing oriented boundaries partially extends to almost minimizing sets. These are boundaries $\partial E$ satisfying the following nearly optimal isoperimetric inequality (9), with area minimizers corresponding to the optimal inequality. Assume there are some $K>0, \beta \in(0,1)$ so that for $\Omega \subset \mathbb{R}^{n+1}$ open, $E \subset$ $\mathbb{R}^{n+1}$, any $x \in \Omega$ and any $\rho \in(0, \operatorname{dist}(x, \partial \Omega))$ :

$$
\int_{B_{\rho}(x)}\left|D \chi_{E}\right| \leq \inf \left\{\int_{B_{\rho}(x)}\left|D \chi_{F}\right| \mid F \Delta E \subset \subset B_{\rho}(x)\right\}+K \cdot \rho^{n+2 \cdot \beta},
$$

where $F \Delta E:=(F \backslash E) \cup(E \backslash F), \chi_{A}$ is the characteristic function of $A \subset \mathbb{R}^{n}$ and $\int_{\Omega}\left|D \chi_{A}\right|:=\sup \left\{\left.\int_{\Omega} \chi_{A} \cdot \operatorname{div} g d \mu\left|g \in C_{0}^{1}\left(\Omega, \mathbb{R}^{n}\right),\right| g\right|_{C^{0}} \leq 1\right\}$ is the perimeter of $A$. Again, these conditions admit obvious generalizations to the Riemannian manifold case.

The following result, extending step 1 in De Giorgi's approach, is due to Tamanini [22], [23]:

Theorem 3.2 ( $C^{1, \beta}$-Regularity) An oriented boundary $\partial E$ satisfying (9) is a $C^{1, \beta}$. hypersurface except for some singular set of Hausdorff-dimension $\leq n-7$. Sequences of infinite rescalings of $\partial E$ flat norm subconverge to area minimizing tangent cones $C^{n} \subset \mathbb{R}^{n+1}$.

As in the area minimizing case we now require an upgrade to $C^{k}$-convergence, $k \geq 2$. Then we may extend the setup for the ID-maps of 3.1 to transfer $C^{k}$-regular functions from $\partial E$ to tangent cones to compare solutions on these two spaces. To this end, we note that this $C^{k}$-convergence implies asymptotic constraints on the mean curvature of $H$ towards $\Sigma_{H}$ since $C^{n} \subset \mathbb{R}^{n+1}$ has vanishing generalized mean curvature ${ }^{2}$ which, in turn, causes the $C^{\infty}$-regularity of $C \backslash \sigma_{C}$. That is, when we seek for oriented boundaries satisfying (9)

\footnotetext{
${ }^{1}$ We simply write ID when all other details are known from the context.

${ }^{2}$ Generalized mean curvatures can be defined, using variational integrals, even for Caccioppoli sets and more commonly, using partial integration, e.g. for varifolds, cf. [3] and [20] for discussions.
} 
with high regularity tangent cone approximations, then mean curvature constraints are the natural candidate.

Example 3.3 (Mean Curvature Problems) We consider two basic examples of oriented boundaries satisfying (9). The first one stems from geometry, the other one from general relativity.

- Hypersurfaces of Prescribed Mean Curvature, in the simplest case we consider here also called $\mu$-bubbles, cf. [14, p.59-61] and [9, Ch.2] for background material. In this case $\mu$ is a smooth function on a Riemannian manifold $M^{n+1}$. An oriented boundary $H^{n}$ locally bounding an open set $H^{+} \subset M^{n+1}$ is called a $\mu$-bubble if it locally minimizes the variational integral

$$
\operatorname{Area}_{n}(H)-\int_{H^{+}} \mu d V_{n+1}
$$

Then $H$ satisfies (9), relative to $M$, and it has a locally bounded generalized mean curvature that equals $\mu$. Due to the ellipticity of $\mu=\operatorname{tr} A_{H}$, [12, Cor.16.7], $H \backslash \Sigma_{H}$ is $C^{\infty}$-regular and the tangent cone approximation in terms of ID-maps is of class $C^{k}$, for any $k \geq 2$.

- Marginally Outer Trapped Surfaces, abbreviated MOTS, are models for horizons of black holes [19]. The setup is that $M^{n+1}$ is a Riemannian manifold considered as a Cauchy initial hypersurface in some Lorentzian manifold $L^{n+2}$. The horizon $H$ of a black hole is the outermost hypersurface $H^{n} \subset M^{n+1}$ that does not necessarily collapse into the black hole that $H$ surrounds, while evolving in $L$. The defining condition is that $H$ balances the mean curvature $\operatorname{tr}_{H} A_{H}$ of $H$ in $M$ against the (partial) mean curvature $\operatorname{tr}_{H} A_{M \mid H}$ of $M$ in $L$ evaluated on $H$ :

$$
\operatorname{tr}_{H} A_{M \mid H}+\operatorname{tr}_{H} A_{H}=0
$$

Remarkably, MOTS are not known to arise from variational principles. Instead the existence proof employs a less common blow-up method for Jang's equation on $M$ controlled by Perron type arguments [8]. The resulting MOTS $H$ is a boundary that satisfies (9). Again, we infer from (11) and elliptic theory that $H \backslash \Sigma_{H}$ is $C^{\infty}$-regular and the tangent approximation is of class $C^{k}$, for any $k \geq 2$, cf. [8, Appendix].

Remark 3.4 (Currents) In the literature, cf. [9], one also considers almost minimizing currents. Here the decomposition into oriented boundaries is less helpful since there is no strict maximum principle and ID-representations using tangent cones become more subtle due to the presence of points of higher density. On the other hand, the local oriented boundary case covers typical applications in geometry and physics, e.g. those in 3.3 above. That said, a future direction clearly is to find appropriate extensions of hyperbolic unfoldings and their applications to potential theory to currents, varifolds and the vastly more difficult case of higher codimension.

This discussion suggests a definition for almost minimizers tailored to cover also cases with a regularity theory we cannot trace back to standard procedures like variational principles. We use the ID-maps setup of 3.1 as a blueprint and consider oriented boundaries in the sense that there are local representations as boundaries of open subsets of the ambient space: 
Definition 3.5 (Almost Minimizers and ID-Maps) An oriented boundary $H^{n} \subset M^{n+1}$ satisfying the isoperimetric inequalities (9) in any $p \in H$ is called a $C^{k, \gamma}$-tame almost minimizer, for $k \geq 2, \gamma \in(0,1)$, provided the following conditions hold:

- The (generalized) mean curvature is locally bounded.

- $H \backslash \Sigma$ and the ID-maps $\Gamma_{i}$ in 3.1 are $C^{k, \gamma}$-regular with $\left|\mathbf{I D}-i d_{C}\right|_{C^{k, \gamma}\left(B_{R}(q) \cap C\right)} \rightarrow 0$, for $i \rightarrow \infty$, for any given tangent cone $C, p \in \Sigma_{H}$ and $q \in C$ with $\overline{B_{R}(q)} \subset C \backslash \sigma_{C}$, for some $R>0$.

When we say $H \in \mathcal{G}$ we mean $C^{k, \gamma}$-tame almost minimizer for some $k \geq 2$ and $\gamma \in$ $(0,1)$. To simplify the presentation we generally assume $C^{k, \gamma}$-tameness for any $k$.

Remark 3.6 (Examples and Variations) (i) The two sample cases we discussed in 3.3 and, with the local decomposition into disjoint oriented boundaries, cf.[11, Theorem 7 in 4.3.1], any area minimizer $H \in \mathcal{H}_{n}$ is $C^{k, \gamma}$-tame for any $k \geq 2, \gamma \in(0,1)$.

(ii) Since the claimed properties hold for any $B_{R}(q)$, the same assertions extends to any bounded open set $U$ with $\bar{U} \subset C \backslash \sigma_{C}$ using finite ball covers.

(iii) The elliptic operators $L$ on $H \in \mathcal{G}$ we consider in this paper have at least $C^{\alpha}$-regular coefficients, for some $\alpha \in(0,1)$. To draw the typical conclusions, for instance that solutions of $L \phi=0$ are $C^{2, \alpha}$-regular, we choose $k+\gamma$ large enough, let us say $k=5$, to be on the safe side.

(iv) When we do not fix the base point $p \in \Sigma_{H}$, but move it along $\Sigma$ during the scaling by $\tau_{i}$, the subconvergence under blow-ups may lead to a general area minimizing oriented Euclidean boundary $H^{*}$ with the second condition reading the same with $C$ replaced by $H^{*}$.

Tangent Cone Freezing In the classical interpretation of tangent cones, blowing up around $p \in \Sigma_{H}$, for $H \in \mathcal{G}$, merely yields subconvergence to some not uniquely determined tangent cone. There is a simple but quite effective way to revamp this picture. We recall some concepts from [17, Chapter 4.1]. We choose some $\omega>0$ and consider the $\mathcal{S}$-pencil $\mathbb{P}(p, \omega)$ pointing to $p \in \Sigma$ :

$$
\mathbb{P}(p, \omega)=\mathbb{P}_{H}(p, \omega)=\left\{x \in H \backslash \Sigma_{H} \mid \delta_{\langle A\rangle_{H}}(x)>\omega \cdot d_{H}(x, p)\right\} .
$$

It is useful to also define the truncated $\mathcal{S}$-pencils $\mathbb{T P}$. The $\mathbb{T P}$ are in controllable distance to the singular set and ID-maps easily extend to these sets.

$$
\mathbb{T P}(p, \omega, R, r)=\mathbb{T P}_{H}(p, \omega, R, r):=B_{R}(p) \backslash B_{r}(p) \cap \mathbb{P}(p, \omega) \subset H .
$$

While we scale using increasingly large $\tau>0$, we observe that $\tau \cdot \mathbb{T P}_{H}(p, \omega, R / \tau, r / \tau)$ is better and better $C^{k}$-approximated by the corresponding truncated $\mathcal{S}$-pencil in the given tangent cone. Intuitively, the twisting of $\mathbb{P}(p, \omega)$ slows down as $\tau \rightarrow \infty$, it asymptotically freezes.

Proposition $3.7\left(\mathcal{S}\right.$-Freezing) Let $H \in \mathcal{G}$ and $p \in \Sigma_{H}$. Further, pick some $\varepsilon>0$ and $a$ pair $R>1>r>0$. Then the following is true.

- Flat-norm Form: We have some $\tau_{\varepsilon, R, r, p}>1$ such that for every $\tau \geq \tau_{\varepsilon, R, r, p}$ there is some tangent cone $C_{p}^{\tau}$ in $p$ with $\tau \cdot\left(H \cap B_{R / \tau}(p) \backslash B_{r / \tau}(p)\right) \varepsilon$-close in flat norm to $C_{p}^{\tau} \cap$ $B_{R}(0) \backslash B_{r}(0)$. 
- Smooth Form: For any additional $\omega \in(0,1)$, we can find $\tau_{\varepsilon, R, r, \omega, p} \geq \tau_{\varepsilon, R, r, p}$ such that for every $\tau \geq \tau_{\varepsilon, R, r, \omega, p}$ the rescaled $\tau \cdot \mathbb{T P}(p, \omega, R / \tau, r / \tau) \subset \tau \cdot H$ can be written as a smooth section $\Gamma_{\tau}$ with $\left|\Gamma_{\tau}\right|_{C^{k, \gamma}}<\varepsilon$ of the normal bundle of $C_{p}^{\tau}$ in $\mathbb{R}^{n+1}$ over $\mathbb{T P}(0, \omega, R, r) \subset C_{p}^{\tau}$.

Proof Assume that the required $\tau_{\varepsilon, R, r, p}$ does not exist. Then there is a sequence $\tau_{i} \rightarrow \infty$, $i \rightarrow \infty$ such that none of the $\tau_{i} \cdot\left(H \cap B_{R / \tau_{i}}(p) \backslash B_{r / \tau_{i}}(p)\right)$ is $\varepsilon$-close in flat norm to $C \cap B_{R}(0) \backslash B_{r}(0)$ for any tangent cone $C$. However, from Thm. 3.2 we know that $\tau_{i} \cdot H$ is subconvergent to some tangent cone $C$ and there exists a sufficiently large $i_{0}$ with $\tau_{i_{0}} \cdot\left(H \cap B_{R / \tau_{i_{0}}}(p) \backslash B_{r / \tau_{i_{0}}}(p)\right) \varepsilon$-close to $C^{*} \cap B_{R}(0) \backslash B_{r}(0)$ for some tangent cone $C^{*}$, contradicting the assumption.

For the smooth form assume there is no $\tau_{\varepsilon, R, r, \omega, p}$ as asserted. Then there is a sequence $\tau_{i} \rightarrow \infty, i \rightarrow \infty$, such that $\tau_{i} \cdot H$ can not be written locally as a section $\Gamma_{\tau_{i}}$ with $\left|\Gamma_{\tau_{i}}\right|_{C^{k, \gamma}}<\varepsilon$ of the normal bundle over $\mathbb{T P}(0, \omega, R, r) \subset C_{p}$ for some tangent cone $C_{p} \in \mathcal{S C}_{n}$. However, by the compactness of $\mathcal{S C}_{n}$ in flat norm topology, cf. [17, Lemma 4.3], and the flat norm part of the assertions just established, the sequence of truncated pencils $\mathbb{T P}(0, \omega, R, r) \subset C_{p}^{\tau_{i}}$ subconverges in flat norm to $\mathbb{T P}(0, \omega, R, r) \subset C$ for some tangent cone $C \in \mathcal{S C}_{n}$. This implies $C^{k, \gamma}$-subconvergence of $\tau_{i} \cdot H$, from 3.5, again a contradiction.

\subsection{Induced Solutions}

In this and the next section we extend the $\mathcal{S}$-freezing results for the asymptotic geometry of $H$ to similar results for the asymptotic analysis of operators $L(H)$ on $H$ that we may naturally associate to (almost) area minimizers $H$. The geometric operators of Ch. 2.4 are typical examples of such natural operators. The main purpose of the present section is to properly define the concept of solutions $L(C) \phi=0$ on tangent cones $C$ that are induced from a given solution of $L(H) \phi=0$ on $H$. In the next section we see that minimal growth properties are handed down from a solution on $H$ to the induced solutions on tangent cones.

Definition 3.8 (Natural Assignments) An assignment $F: H \mapsto F_{H}, H \in \mathcal{G}$, of functions $F_{H}: H \backslash \Sigma_{H} \rightarrow \mathbb{R}$ is called natural if $F_{H}$ commutes with the convergence of underlying spaces. More precisely, for any pointed sequence $H_{i} \in \mathcal{G}, p_{i} \in H_{i} \backslash \Sigma_{H_{i}}$ that is locally converging in flat norm to a pointed space $H, p \in H \backslash \Sigma_{H}$, there is a neighborhood $U(p) \subset$ $H \backslash \Sigma_{H}$ such that

$$
\left|\mathbf{I D}^{*} F_{H_{i}}-F_{H}\right|_{C^{m, \theta}(U(p))}=\left|F_{H_{i}} \circ \mathbf{I D}-F_{H}\right|_{C^{m, \theta}(U(p))} \rightarrow 0 \text { as } i \rightarrow \infty
$$

for some $m(F) \geq 0, \theta(F) \in(0,1)$.

This naturality concept extends to assignments $H \mapsto F_{H}$ of tensors or operators defined over $H \backslash \Sigma$. In the latter case we refer to the operator $F(H)$ itself as natural. To formally establish or to apply this naturality one may choose representations in terms of tuples of functions $\left(F_{1}, \ldots, F_{k}\right)$ and local coordinates, with $m\left(F_{i}\right) \geq 0, \theta\left(F_{i}\right) \in(0,1)$.

We are interested in properties of natural operators that are stable under convergence, for instance under blowing up. As a first example we prove that (shifted) $\mathcal{S}$-adaptedness is stable. 
Lemma 3.9 (Inherited $\mathcal{S}$-Adaptedness) Let $L$ be a natural operator and $H \in \mathcal{G}$. Moreover, assume that $L(H)$ is (shifted) $\mathcal{S}$-adapted. Then $L(N)$ is (shifted) $\mathcal{S}$-adapted on any blow-up $N$ of $H$ around a singular point in $\Sigma \subset H$ and the principal eigenvalues satisfy $\lambda_{L, H}^{\langle A\rangle} \leq \lambda_{L, N}^{\langle A\rangle}$.

Proof For any smooth function $f$ with compact support $K \subset N \backslash \Sigma_{N}$ we choose a sufficiently large scaling factor $\eta \gg 1$ such that the ID-map between $\eta \cdot H$ and $N$ in a neighborhood of $K$ is very close to an isometry in $C^{k, \gamma}$-topology. Then the naturality of $L$ and $\langle A\rangle$ implies that

$$
\int_{H} f \circ \mathbf{I D}^{-1} \cdot L(\eta \cdot H)\left(f \circ \mathbf{I D}^{-1}\right) d V / \int_{H}\langle A\rangle_{H}^{2} \cdot f^{2} \circ \mathbf{I D}^{-1} d V
$$

is arbitrarily close to

$$
\int_{N} f \cdot L(N)(f) d V / \int_{N}\langle A\rangle_{N}^{2} \cdot f^{2} d V
$$

upon choosing $\eta$ large enough. Thus the eigenvalue of $\delta_{\langle A\rangle}^{2} \cdot L(H)$ on $H$ lower estimates the second integral. In particular, the Hardy inequality holds for $L(N)$ on $N$. The adaptedness property of $L(N)$ follows from the scaling invariance of the estimates and the adaptedness of $L(H)$.

In the following discussion we choose some natural (shifted) $\mathcal{S}$-adapted operator $L$ and consider solutions $u>0$ of $L \phi=0$ either on bounded subsets $U \subset H \backslash \Sigma_{H}$ or on the whole regular set $H \backslash \Sigma_{H}$. In the latter case we say $u$ is an entire solution.

Proposition 3.10 ( $\mathcal{S}$-Freezing of Solutions) Let $H \in \mathcal{G}$ and $p \in \Sigma_{H}$. Further, pick some $\varepsilon>0$ and a pair $R>1>r>0$ and some $\omega \in(0,1)$ and an entire solution $u>0$ of $L(H) \phi=0$. Then there exists some $\tau^{*}(L, u, \varepsilon, \omega, R, r, p)>0$ such that for any $\tau \geq \tau^{*}$ the following is true:

There is some tangent cone $C_{p}^{\tau}$ with $\left|\mathbf{I D}-i d_{C_{p}^{\tau}}\right|_{C^{k, \gamma}\left(\mathbb{T P}_{C_{p}^{\tau}}(0, \omega, R, r)\right)} \leq \varepsilon$, from 3.7, and there is an entire solution $v>0$, of $L\left(C_{p}^{\tau}\right) \phi=0$, that can be chosen independently of $\varepsilon, \omega, R, r$, with

$$
|u \circ \mathbf{I D} / v-1|_{C^{2, \alpha}\left(\mathbb{T P}_{C_{p}^{\tau}}(0, \omega, R, r)\right)} \leq \varepsilon .
$$

We call such an entire solution $v$ on $C_{p}^{\tau}$ an induced solution.

Completely similarly we get induced solutions on more general blow-up hypersurfaces in $\mathcal{H}_{n}^{\mathbb{R}}$ obtained from scaling an almost minimizer $H \in \mathcal{G}$ without fixing a basepoint. The reason why we call this a $\mathcal{S}$-freezing of solutions is that, via ID-maps, the solution $u$ can be approximated by $v$ on gradually larger subsets of $C \backslash \Sigma_{C}$ and, in the case of minimal growth constraints, this solution $v$ actually is uniquely determined up to multiples, cf. Ch. 3.3, and it admits a separation of variables, cf. Thm. 4.4. These are basic pieces of the inductive asymptotic analysis of $L$ on $H$.

Proof We argue in two steps. We first show for any sequence $\tau_{i} \rightarrow \infty$, for $i \rightarrow \infty$, after selecting a subsequence, there is a tangent cone $C$ so that, after some normalization, $u \circ \Gamma_{i}$ 
converges compactly to some entire solution on $C$. Then following an argument similar to the case of geometric $\mathcal{S}$-freezing 3.7, we use this to show the claimed stronger assertion.

Step 1 We start with an arbitrary sequence $\tau_{i} \rightarrow \infty$, for $i \rightarrow \infty$. After selecting a subsequence there is a tangent cone $C$ so that $\tau_{i} \cdot H$ converges to $C$ as described in 3.1. Due to the naturality of $L$, the ID-pull-back of $L\left(\tau_{i} \cdot H\right)$, expressed in terms of coefficients, compactly converges to $L(C)$ for $i \rightarrow \infty$. Thus, for the solution $u$ of $L(H) \phi=0$ we observe that the $u \circ \Gamma_{i}$ eventually solve an elliptic equation on any given common domain $U$ with compact closure in $C \backslash \sigma_{C}$ whose coefficients are arbitrarily close in Hölder norm to those of $L(C)$. From this we get uniform constants for elliptic regularity estimates and Harnack inequalities valid for any of these pull-back operators on $U, \mathrm{cf}$. [4, 7] or [12]. Now we choose the normalization

$$
u_{i}:=u \circ \Gamma_{i} / u \circ \Gamma_{i}(q) \text {, for some basepoint } q \in U .
$$

Then, we can employ the (uniform) Harnack inequalities and the compactness of the embedding $C^{2, \alpha} \subset C^{2, \beta}$, for $\beta \in(0, \alpha)$, on $U$, to see that there is a subsequence compactly $C^{2, \beta}$-converging to some positive and, via elliptic theory, again $C^{2, \alpha}$-solution of $L(C) \phi=0$ on $U$. Choosing an exhaustion $U_{l} \subset U_{l+1}$ $l \in \mathbb{Z}^{\geq 1}, q \in U_{1}$, with $\bigcup_{l} U_{l}=C \backslash \sigma_{C}$ by such domains $U_{l}$ and choosing diagonal sequences we infer that the $u_{i}$ compactly subconverge to some positive entire solution $u_{\infty}$ of $L(C) \phi=0$ on $C \backslash \sigma_{C}$.

Step 2 Assume that for some $\varepsilon>0, R>1>r>0, \omega>0$, there exists a sequence $\tau_{i} \rightarrow \infty$, for $i \rightarrow \infty$, such that for any entire solution $v>0$ of $L(C) \phi=0$ on any tangent cone $C \backslash \sigma_{C}$ we have $\left|u \circ \Gamma_{i} / v-1\right|_{C^{2, \alpha}\left(\mathbb{T P}_{C}(0, \omega, R, r)\right)} \geq \varepsilon$. Compactness of the space of tangent cones $\mathcal{T}_{p}$ in $p$ [17, Lemma 4.3] gives a subsequence $\tau_{i_{k}}$ and cones $C_{p}^{\tau_{i_{k}}}$ converging to some tangent cone $C_{p}$. Then we get ID-maps $\Gamma_{i_{k}}^{*}$ representing open subsets of $C_{p}^{\tau_{i_{k}}} \backslash \sigma_{C_{p}} \tau_{i_{k}}$ as section of the normal bundle over $C_{p} \backslash \sigma_{C_{p}}$ converging to the zero section. For sufficiently large $k$ we choose the normalization

$$
u_{i_{k}}:=u \circ \Gamma_{i_{k}} \circ \Gamma_{i_{k}}^{*} / u \circ \Gamma_{i_{k}} \circ \Gamma_{i_{k}}^{*}(q) \text {, for some } q \in C_{p} \backslash \sigma_{C_{p}} .
$$

From Step 1 we can now find another subsequence $\tau_{i_{k_{m}}}$ of $\tau_{i_{k}}$ such that the normalized $u_{i_{k_{m}}}$ induce a positive entire solution on $C_{p}$ contradicting the assumption. Thus we get an entire solution $v_{\tau}^{*}(\varepsilon, \omega, R, r)>0$ satisfying (12) for some $C_{p}^{\tau}$. For $\varepsilon, \omega, R^{-1}, r \rightarrow 0$ we repeat these compactness and contradiction arguments to see that, for $i$ large enough, there is a solution $v>0$ satisfying (12) that is also independent of these parameters.

The argument for 3.10 also shows that entire solutions on converging sequences of singular cones induce entire solutions on the limit cone.

Corollary 3.11 (Induced Solutions on $\mathcal{S C}_{n}$ ) Let $\varepsilon, \omega>0$ and $R>1>r>0$. Then there exists a $\zeta(L, \varepsilon, \omega, R, r)>0$ such that following holds: For any $C$ and $C^{\prime} \in \mathcal{C}_{n}$ with $d_{H}\left(\partial B_{1}(0) \cap C, \partial B_{1}(0) \cap C^{\prime}\right) \leq \zeta$ we have $\left|\mathbf{I D}-i d_{C}\right|_{C^{k, \gamma}\left(\mathbb{P P}_{C}(0, \omega, R, r)\right)} \leq \varepsilon$, and for any entire solution $u_{C^{\prime}}>0$ of $L\left(C^{\prime}\right) \phi=0$ there is an entire solution $u_{C, C^{\prime}}>0$ of $L(C) \phi=0$ 
that may be chosen independently of the parameters $\varepsilon, \omega, R$ and $r$ with

$$
\left|u_{C^{\prime}} \circ \mathbf{I D} / u_{C, C^{\prime}}-1\right|_{C^{2, \alpha}\left(\mathbb{T P}_{C}(0, \omega, R, r)\right)} \leq \varepsilon .
$$

Again, we call such an entire solution $u_{C, C^{\prime}}$ on $C$ an induced solution.

Tangent cones may not be unique but this result, when combined with stability results for minimal growth constraints, extends compactness results for cone spaces to their minimal growth solutions, cf. 3.13. This implies, for instance, common growth estimates for such solutions on all tangent cones in a given singular point.

\subsection{Minimal Growth Stability}

Let $L$ be a natural $\mathcal{S}$-adapted Schrödinger operator $L$. We show that $L$-vanishing is a stable property which persists under deformations of the underlying spaces. A far reaching consequence is that any positive solution of $L \phi=0$ that is $L$-vanishing around some singular $p \in \Sigma_{H}$ induces solutions on any tangent cone $C$ at $p$ that are $L$-vanishing towards the entire singular set $\sigma_{C}$. In other words, the asymptotic minimal growth of solutions and the convergence of the underlying spaces are interchangeable. This is a remarkable phenomenon as the singular set of the underlying space, and thus the meaning of $L$-vanishing, may change dramatically under deformations.

Theorem 3.12 (Blow-Up Stability of $L$-Vanishing Properties) Let $H \in \mathcal{G}$ and let $L$ be some natural, $\mathcal{S}$-adapted Schrödinger operator on $H \backslash \Sigma$. Further, let $u>0$ be a solution of $L \phi=0$ that is $L$-vanishing in a neighborhood $V$ of some point $p \in \Sigma$. Then we have the following inheritance results:

- If $C$ is a tangent cone of $H$ in p, then any solution induced on $C$ is L-vanishing along $\sigma_{C}$.

- $\quad$ More generally, for a sequence $s_{i} \rightarrow \infty$ of scaling factors and a sequence of points $p_{i} \rightarrow p$ in $\Sigma_{H}$ such that $\left(s_{i} \cdot H, p_{i}\right)$ subconverges to a limit space $\left(H_{\infty}, p_{\infty}\right)$ with $H_{\infty} \in \mathcal{H}_{n}^{\mathbb{R}}$, any induced solution on $H_{\infty} \backslash \Sigma_{H_{\infty}}$ L-vanishes along $\Sigma_{\infty}$.

More generally, [17, Theorem 3.4] shows that it suffices when $u$ is a supersolution on $H \backslash \Sigma$ and a solution on $V$ since the boundary Harnack inequality applies in this case as well.

The Martin theory for these limit spaces says that there is, up to multiples, precisely one positive solution $L$-vanishing along the singular set $\Sigma_{H_{\infty}}$. It is the unique and minimal Martin boundary point at infinity $\infty_{H_{\infty}}$, cf. [17, Theorem 3]. We write this solution as $\Psi_{+}=$ $\Psi_{+}\left(H_{\infty}, L\right)$. Similarly, for any cone $C \in \mathcal{S C}_{n}$, there is also a unique (minimal) Martin boundary point at the origin $0_{C}$, the positive solution $\Psi_{-}=\Psi_{-}(C, L)$ that is $L$-vanishing along $\sigma_{C} \cup\left\{\infty_{C}\right\} \backslash\left\{0_{C}\right\}$.

A basic problem with tangent cones in singular points is that they are generally nonunique. The following second stability theorem partial compensates this issue. We show that the induced solutions change continuously when we move from one to another tangent cone. Therefore we can extend compactness results for area minimizers to the assigned functions $\Psi_{+}$. This helps to derive a variety of uniform estimates for such solutions for all cones in $\mathcal{S C}_{n}$ 
Theorem 3.13 (Stable $L$-Vanishing on Cones) Let $L$ be a natural and $\mathcal{S}$-adapted Schrödinger operator on cones $C \in \mathcal{S C}_{n}$. Then, for any flat norm converging sequence $C_{i} \rightarrow C_{\infty}, i \rightarrow \infty$, with appropriately normalized associated solutions $\Psi_{ \pm}\left(C_{i}\right)$ and $\Psi_{ \pm}\left(C_{\infty}\right)$, we have

$$
\Psi_{ \pm}\left(C_{i}\right) \circ \mathbf{I D} \rightarrow \Psi_{ \pm}\left(C_{\infty}\right) C^{2, \alpha} \text {-compactly on } C_{\infty} \backslash \sigma_{C_{\infty}} \text { as } i \rightarrow \infty
$$

A more general version of this result applies to $\mathcal{H}_{n}^{\mathbb{R}}$. A particularly interesting special case are degenerating sequences of regular Euclidean hypersurfaces $H_{i} \in \mathcal{H}_{n}^{\mathbb{R}}$ with singular limit. Then the induced solutions are always $L$-vanishing along the singular set of the limit hypersurface.

Theorem 3.14 (Stable $L$-Vanishing on $\mathcal{H}_{n}^{\mathbb{R}}$ ) Let $H_{i} \in \mathcal{H}_{n}^{\mathbb{R}}$ be a compactly converging sequence, $i \geq 1$, with limit $H_{\infty} \in \mathcal{H}_{n}^{\mathbb{R}}$. Further, let $L=-\Delta+V$ be a natural, $\mathcal{S}$-adapted Schrödinger operator with $\lambda_{L, H_{i}}^{\langle A\rangle} \geq c$ for some $c>0$ independent of $i$ and $u_{i}>0$ solutions of $L \phi=0$ on $H_{i} \backslash \Sigma_{H_{i}}$. Now assume the $u_{i}$ are $L$-vanishing along $U \cap \Sigma_{H_{i}}$, for some open $U \subset \mathbb{R}^{n+1}$. Then any induced solution $u>0$ on $H_{\infty}$ also L-vanishes along $U \cap \Sigma_{H_{\infty}}$.

The proofs of 3.12-3.14 occupy the remainder of this section. The arguments for these results essentially coincide and will be addressed simultaneously. We first derive variants 3.16 of these results for minimal Green's functions which are $L$-vanishing along the entire singular set. This way we separate the invariance of $L$-vanishing properties from the problem to work with the localization that the given solution $u>0$ of $L \phi=0$ is $L$-vanishing only in a neighborhood $U$ of some point $p \in \Sigma$. This localization is considered in a second step. This is another occasion where we employ the boundary Harnack inequalities already used to ensure the uniqueness of $\Psi_{ \pm}$.

In these arguments we use some functional analytic consequences of $\mathcal{S}$-adaptedness. We show that the Hardy inequality extends from $C_{0}^{1}(H \backslash \Sigma)$ to $H_{\langle A\rangle}^{1,2}(H \backslash \Sigma)$ and that furthermore, $\mathcal{S}$-adaptedness also allows us to control the $H_{\langle A\rangle}^{1,2}$-norm, see [17, Ch.5.1].

Lemma 3.15 (Equivalence of Norms) Let $L=-\Delta+V$ be a natural, $\mathcal{S}$-adapted Schrödinger operator with principal eigenvalue $\lambda_{L, H}^{\langle A\rangle}>0$ for some $H \in \mathcal{G}$. Then there are constants $\beta_{L, H}^{\langle A\rangle}$ and $\beta_{L, H}^{\langle A\rangle, *} \geq 1$ such that

$$
\begin{aligned}
\beta_{L, H}^{\langle A\rangle} \cdot|f|_{H_{\langle A\rangle}^{1,2}(H \backslash \Sigma)} & \geq \int_{H \backslash \Sigma} f \cdot L f d V \geq \lambda_{L, H}^{\langle A\rangle} \cdot \int_{H \backslash \Sigma}\langle A\rangle^{2} \cdot f^{2} d V \\
\beta_{L, H}^{\langle A\rangle, *} \cdot \int_{H \backslash \Sigma} f \cdot L f d V & \geq|f|_{H_{\langle A\rangle}^{1,2}(H \backslash \Sigma)} f \cdot
\end{aligned}
$$

for any $f \in H_{\langle A\rangle}^{1,2}(H \backslash \Sigma)$.

Proof We recall that $\langle A\rangle$-adaptedness means that for some $a_{L}>0$ the potential $V$ satisfies

$$
-a_{L} \cdot\langle A\rangle^{2} \leq V \leq a_{L} \cdot\langle A\rangle^{2} .
$$

Therefore, [17, Theorem 5.3] shows that the inequality $\int_{H \backslash \Sigma} f \cdot L f d V \geq \lambda_{L, H}^{\langle A\rangle} \cdot \int_{H \backslash \Sigma}\langle A\rangle^{2}$. $f^{2} d V$ not only holds for test functions in $C_{0}^{1}(H \backslash \Sigma)$ but actually for all functions in $H_{\langle A\rangle}^{1,2}(H \backslash \Sigma)$. This implies the first inequality (16) on $H_{\langle A\rangle}^{1,2}(H \backslash \Sigma)$. 
For the second inequality we use Eq. 18 again to write

$$
\begin{gathered}
\left(1+\left(a_{L}+1\right) / \lambda_{L, H}^{\langle A\rangle}\right) \cdot \int_{H \backslash \Sigma} f \cdot L f d V \\
\int_{H \backslash \Sigma}\left(\left|\nabla_{H} f\right|^{2}+V \cdot f^{2}\right) d V+\left(a_{L}+1\right) \cdot \int_{H \backslash \Sigma}\langle A\rangle^{2} \cdot f^{2} d V \geq|f|_{H_{\langle A\rangle}^{1,2}(H \backslash \Sigma)}^{\geq}
\end{gathered}
$$

whence Eq. 17 with $\beta_{L, H}^{\langle A\rangle, *}:=1+\left(a_{L}+1\right) / \lambda_{L, H}^{\langle A\rangle}$. The inequality (*) uses a partial integration valid for all $f \in C_{0}^{1}(H \backslash \Sigma)$. To extend (*) to $H_{\langle A\rangle}^{1,2}(H \backslash \Sigma)$ we choose any $f \in H_{\langle A\rangle}^{1,2}(H \backslash \Sigma)$ and show that $\int_{\partial U_{r_{i}}}|f| \cdot|\nabla f| d A \rightarrow 0$, for $U_{r}:=\left\{x \in H \backslash \Sigma \mid \delta_{\langle A\rangle}(x)<r\right\}$, for some sequence $r_{i} \rightarrow 0$. To this end we use the coarea formula [11, 2.1.5, Theorem 3] and (S3): $\left|\delta_{\langle A\rangle_{H}}(p)-\delta_{\langle A\rangle_{H}}(q)\right| \leq L_{\langle A\rangle} \cdot d_{H}(p, q)$ and get:

$\int_{0}^{1}\langle A\rangle \int_{\partial U_{r}}|f| \cdot|\nabla f| d r d A \leq L_{\langle A\rangle} \cdot \int_{U_{1}}\langle A\rangle \cdot|f| \cdot|\nabla f| d V \leq 2 \cdot L_{\langle A\rangle} \cdot \int_{H \backslash \Sigma}\left|\nabla_{H} f\right|^{2}+\langle A\rangle^{2} \cdot f^{2} d V$

Moreover, we have $\langle A\rangle(x) \geq L_{\langle A\rangle}^{-1} \cdot \operatorname{dist}(x, \Sigma)^{-1}$ from (S2) and (S2). Therefore we find that $\int_{\partial U_{r}}|f| \cdot|\nabla f| d A$ cannot be positively lower bounded for $r \in(0,1]$, i.e. there is a sequence $r_{i} \rightarrow 0$ so that $\int_{\partial U_{r_{i}}}|f| \cdot|\nabla f| d A \rightarrow 0$.

The subsequent theorem asserts that minimality of Green's functions $G(x, y)$ is stable under perturbation of the underlying area minimizer. In the argument we show that $G(x, y)$ can be described as finite energy minimizers of a Dirichlet type integral outside regular balls $B_{\rho}(y)$ centered around the pole $y$ of $G(\cdot, y)$. By the previous lemma this also entails finite $H_{\langle A\rangle}^{1,2}$-norm. This is by no means obvious since in general, even minimal growth solutions strongly diverge towards $\Sigma$ and general solutions usually lead to an infinite $H_{\langle A\rangle}^{1,2}$-norm, cf. Theorem 4.4 and Proposition 4.5 below for growth estimates.

We consider two situations of converging hypersurfaces using ID-map identifications.

$\mathrm{S}_{1} \quad$ Let $H_{i} \in \mathcal{H}_{n}^{\mathbb{R}}, i \geq 1$, be compactly converging, with basepoints $a_{i} \in H_{i} \backslash \Sigma_{H_{i}}$ and possibly with $\Sigma_{H_{i}}=\emptyset$. Let $H_{\infty} \in \mathcal{H}_{n}^{\mathbb{R}}$. be the limit with basepoint $a_{\infty}=\lim _{i \rightarrow \infty} a_{i}$, $d\left(a_{\infty}, 0\right)=\operatorname{dist}\left(a_{\infty}, \Sigma_{H_{\infty}}\right)=5$ and $0 \in \Sigma_{H_{\infty}} \subset H_{\infty}$. Further, let $L=-\Delta+V$ be a natural, $\mathcal{S}$-adapted Schrödinger operator with $\lambda_{L, H_{i}}^{\langle A\rangle} \geq c$ for some $c>0$ independent of $i$.

$\mathrm{S}_{2} \quad$ Let $H \in \mathcal{G}^{c}$ and $H_{i}=\tau_{i} \cdot H \in \mathcal{G}^{c}$, for some sequence $\tau_{i} \rightarrow \infty$. Also we choose basepoints $a_{i} \in H \backslash \Sigma_{H}, i \geq 1$. We assume the $H_{i}$ and $a_{i}$ converge to some limit $H_{\infty} \in \mathcal{H}_{n}^{\mathbb{R}}$ with basepoint $a_{\infty}=\lim _{i \rightarrow \infty} a_{i}, d\left(a_{\infty}, 0\right)=\operatorname{dist}\left(a_{\infty}, \Sigma_{H_{\infty}}\right)=5$ and $0 \in \Sigma_{H_{\infty}} \subset H_{\infty}$. Further, let $L=-\Delta+V$ be a natural, $\mathcal{S}$-adapted Schrödinger operator on $H$.

The reason why we only consider the case of blow-ups of one given almost minimizer $H \in \mathcal{G}^{c}$ is to ensure uniform control over the convergence and naturality properties of $\langle A\rangle$.

Proposition 3.16 (Stability of Minimal Green's Functions) We assume we are in one of the two situations $S_{1}$ and $S_{2}$. We denote by $G_{i}=G_{i}\left(\cdot, a_{i}\right)>0$ the minimal Green's function on $H_{i}$ with pole in $a_{i}$ and normalized to $\int_{\partial B_{1}\left(a_{i}\right)} G_{i}=1$, where $i \geq 1$ or $i=\infty$. Then we have for $i \rightarrow \infty$ :

$$
G_{i} \rightarrow G_{\infty} \quad C^{2, \alpha} \text {-compactly on } H_{\infty} \backslash\left(\Sigma_{H_{\infty}} \cup\left\{a_{\infty}\right\}\right) \text { via ID-maps. }
$$


Proof We focus on case $S_{1}$. Case $S_{2}$ then follows along similar lines noting that the scaling invariance of the $\langle A\rangle^{2}$-weighted principal eigenvalue implies the condition $\lambda_{L, H_{i}}^{\langle A\rangle} \geq c$ for some constant $c>0$ from the $\mathcal{S}$-adaptedness of $L$.

It is a trivial fact that the limit of the $G_{i}$ is again a Green's function; the point is to show its minimality. The idea is to characterize a minimal Green's function $G(\cdot, p)$ on $H$, outside some neighborhood $U$ of the pole $p \in H \backslash \Sigma_{H}$, as the minimizer of the following Dirichlet type integral

$J_{H \backslash U}(f):=\int_{H \backslash U}\left|\nabla_{H} f\right|^{2}+V \cdot f^{2} d V, f$ smooth with supp $f \subset H \backslash \Sigma_{H}$ and $\left.f\right|_{\partial U}=G$.

$\mathcal{S}$-adaptedness of $L$ implies then that the minimality of the $J$-integral is preserved under convergence of the underlying hypersurfaces.

In our situation where we have a compactly converging sequence of pointed minimizers $H_{i} \rightarrow H_{\infty}$ with $a_{i} \rightarrow a_{\infty}$ we set $U_{i}=\mathbf{I D}\left(B_{1}\left(a_{\infty}\right)\right)$ and $U_{\infty}=B_{1}\left(a_{\infty}\right)$. Without loss of generality we may also assume that $B_{5}\left(a_{i}\right) \subset H_{i} \backslash \Sigma_{H_{i}}$, since $B_{5}\left(a_{\infty}\right) \subset H_{i} \backslash \Sigma_{H_{\infty}}$, and these balls are, possibly after rescaling, diffeomorphic and uniformly almost isometric in $C^{3}$-norm to the Euclidean ball $B_{5}(0)$.

The proof will be divided into three steps. In the first two preliminary steps we derive results needed in the third and main step. In the first step we show that the functionals $J_{H_{i} \backslash U_{i}}, i \geq 0$ or $i=\infty$, are uniformly bounded. In Step 2 we shall prove that the $G_{i}$ are the unique minimizers of the Dirichlet type integral above. In Step 3 we prove that $G_{i}$ converge to a limit solution $G^{*}$ which minimizes the Dirichlet integral on $H_{\infty} \backslash U_{\infty}$. (The uniqueness from Step 2 then implies that $G^{*}=G_{\infty}$.) For this we prove that there is no bubbling-off of negative contributions to $J_{H_{i} \backslash U_{i}}(f)$ before we reach the limit. This will use the $\mathcal{S}$-adaptedness of $L$ and the fact that $H_{\langle A\rangle}^{1,2}(H \backslash \Sigma) \equiv H_{\langle A\rangle, 0}^{1,2}(H \backslash \Sigma)$ proved in [17, Theorem 5.3].

Step 1 For the infima inf $J_{i}$ of $J_{H_{i} \backslash U_{i}}(\cdot), i \geq 0$ or $i=\infty$, on $H_{\langle A\rangle}^{1,2}\left(H_{i} \backslash \Sigma_{H_{i}}\right.$, we have a common constant $\alpha>0$ so that

$$
\mid \text { inf } J_{i} \mid \leq \alpha, \text { for any } i \geq 0 \text { or } i=\infty .
$$

For the lower bound let $f$ be a smooth function with supp $f \subset H_{i} \backslash \Sigma_{H_{i}}$ and $\left.f\right|_{\partial U_{i}}=G_{i}$. We extend $f$ as a smooth function to $F_{i}$ on $U_{i}$. Hence $\left|J_{U_{i}}\left(F_{i}\right)\right| \leq c$ for some uniform constant $c<\infty$. The Hardy inequality for $L$ entails, by Lemma 3.9 , that $J_{H_{i}}\left(F_{i}\right) \geq 0$, whence $J_{H_{i} \backslash U_{i}}(f) \geq J_{H_{i}}\left(F_{i}\right)-c \geq-c$. On the other hand, a fixed test function $f$ supported in $B_{2}\left(a_{\infty}\right)$ gives a uniform upper bound for $J_{H_{\infty} \backslash U_{\infty}}(f)$ on $H_{\infty}$ and thus, via almost isometric ID-map pull-back for sufficiently large $i$, also for $J_{H_{i} \backslash U_{i}}(f)$ on $H_{i}$.

Step 2 For the Dirichlet problem of $J_{H_{i} \backslash U_{i}}(\cdot)$ over $H_{\langle A\rangle}^{1,2}\left(H_{i} \backslash \Sigma_{H_{i}}\right)$ the minimal Green's function $G_{i}$ restricted to $H_{i} \backslash U_{i}$ is the unique minimizer $v>0$ with boundary value $\phi=G_{\infty} \circ$ ID on $\partial U_{i}$.

Remark 3.17 (i) If we work with functions in $H_{\langle A\rangle}^{1,2}$, then the Dirichlet problem is considered in the trace sense, cf. [7, Chapter 5.5 and 6.1]. At any rate, the resulting minimizers are regular and thus solve the problem in the classical sense. 
(ii) The claim follows from the general theory of symmetric semi-bounded operators, cf. Remark 3.18 below. But the following explicit argument may be more satisfactory.

For the uniqueness part, we assume we had two distinct positive solutions $v_{1} \neq v_{2}$ with $J_{H_{i} \backslash U_{i}}\left(v_{1}\right)=J_{H_{i} \backslash U_{i}}\left(v_{2}\right)=\inf J_{H_{i} \backslash U_{i}}$. Regularity theory shows that $v_{1,2}$ are at least $C^{2, \alpha}-$ regular. Upon relabeling $v_{1}$ and $v_{2}$ we can find an $\eta<1$ such that $\eta \cdot v_{1}$ and $v_{2}$ coincide in a non-empty set $C$ outside $\overline{B_{1}(p)}$. The Hopf maximum principle shows that $C$ must be a smooth submanifold where the graphs of $v_{1}$ and $v_{2}$ intersect transversally. Since both functions minimize $J$ we observe that

$$
J_{H_{i} \backslash U_{i}}\left(\min \left\{\eta \cdot v_{1}, v_{2}\right\}\right)=\eta^{2} \cdot \inf J_{H_{i} \backslash U_{i}} \text { or } J_{H_{i} \backslash U_{i}}\left(\max \left\{\eta \cdot v_{1}, v_{2}\right\}\right)=\inf J_{H_{i} \backslash U_{i}} .
$$

Thus $\min \left\{\eta \cdot v_{1}, v_{2}\right\}$ or $\max \left\{\eta \cdot v_{1}, v_{2}\right\}$ also minimizes the functional $J$ and must be smooth. But this contradicts the fact that both $\min \left\{\eta \cdot v_{1}, v_{2}\right\}$ and $\max \left\{\eta \cdot v_{1}, v_{2}\right\}$ are non-smooth along $C$. This proves the uniqueness assertion.

For the existence part we choose a sequence of smoothly and compactly bounded domains $\bar{D}_{m} \subset D_{m+1} \subset H_{i} \backslash \Sigma_{H_{i}}, m \geq 0$, with $\bigcup_{m} D_{m}=H_{i} \backslash \Sigma_{H_{i}}$ and $\bar{U} \subset D_{1}$. There is a unique solution $Q_{i, m}$ for the Dirichlet problem with boundary data $Q_{i, m} \equiv G_{i}$ on $\partial U$ and $Q_{i, m} \equiv 0$ on $\partial D_{m}$ which minimizes the Dirichlet functional. We infer from [17, Theorem 5.3] and the proof of the critical case of [17, Theorem 5.7] that $Q_{i, m} \nearrow G_{i}$ and $J_{H_{i} \backslash U_{i}}\left(Q_{i, m}\right) \searrow \inf J_{H_{i} \backslash U_{i}}$ as well as $J_{H_{i} \backslash U_{i}}\left(Q_{i, m}\right) \searrow J_{H_{i} \backslash U_{i}}\left(G_{i}\right)$. Thus the unique minimizer of $J_{H_{i} \backslash U_{i}}$ is the minimal Green's function.

For any $\eta>0$, comparing the functional over compactly supported test functions on the limit space $H_{\infty}$ with the functional over compactly supported test functions on $H_{i}$ via ID-maps yields an $i_{\eta}$ with

$$
J_{H_{i} \backslash U_{i}}\left(G_{i}\right) \leq \eta+J_{H_{\infty} \backslash U_{\infty}}\left(G_{\infty}\right) \text { for any } i \geq i_{\eta} .
$$

This, in turn, implies that

$$
\left|G_{i}\right|_{H_{\langle A\rangle}^{1,2}\left(H_{i} \backslash\left(\Sigma_{H_{i}} \cup U_{i}\right)\right)} \leq \beta \cdot\left(\eta+J_{H_{\infty} \backslash U_{\infty}}\left(G_{\infty}\right)+c^{*}\right),
$$

for some $\beta>0$ independent of $i$ with $\beta_{L, H_{i}}^{\langle A\rangle, *} \geq \beta$, cf. Eq. 17 of Lemma 3.15. $c^{*}>0$ is a common upper bound for the contribution of the extensions of the $G_{i}$ to $U_{i}$ by bounded regular functions. By regularity theory the $G_{i}$ compactly $C^{2, \alpha}$-subconverge to some solution $G^{*}>0$ with $G^{*} \in H_{\langle A\rangle}^{1,2}\left(H_{\infty} \backslash\left(U_{\infty} \cup \Sigma_{H_{\infty}}\right)\right)$ and $G^{*}=G_{\infty}$ on $\partial U$. In the next step we see that $G^{*}$ is uniquely determined, it equals $G_{\infty}$, and, hence, not only a subsequence but the complete sequence $G_{i}$ converges to $G^{*}$.

Step $3 G^{*}$ minimizes the Dirichlet integral whence, $G^{*}=G_{\infty}$.

In view of the compactly $C^{2, \alpha}$-subconverging $G_{i}$, this amounts to exclude bubbling-off phenomena of negative contributions to the Dirichlet integral concentrating near $\Sigma_{i}$ which are no longer visible in the limit. Towards this end, we let $\mathbb{I}_{\varepsilon}:=\left\{x \in H_{i} \backslash \Sigma_{H_{i}} \mid \delta_{\langle A\rangle}(x)<\varepsilon\right\}$, for $\varepsilon>0$ which we choose sufficiently small so that $U_{i} \cap \mathbb{I}_{3 \cdot \varepsilon}=\emptyset$ and $R \geq 10$. Assume that there is a constant $c>0$ independent of both, $\varepsilon$ and $R$, such that there is an $i_{\varepsilon . R}^{0}$ so that

$$
J_{H_{i} \backslash U_{i}}\left(G_{i}\right)=J_{B_{2 \cdot R}\left(a_{i}\right) \backslash\left(U_{i} \cup \mathbb{I}_{\varepsilon}\right)}\left(G_{i}\right)+J_{\left(H_{i} \backslash B_{2 \cdot R}\left(a_{i}\right)\right) \cup \mathbb{I}_{\varepsilon}}\left(G_{i}\right) \text { and } J_{\left(H_{i} \backslash B_{2 \cdot R}\left(a_{i}\right)\right) \cup \mathbb{I}_{\varepsilon}}\left(G_{i}\right) \leq-c
$$

for any $i \geq i_{\varepsilon, R}^{0}$. To argue that this cannot happen we use the setup from the proof of [17, Theorem 5.3]. We first construct a cut-off function concentrated near $\Sigma_{i}$ by taking 
some fixed $\psi \in C^{\infty}(\mathbb{R},[0,1])$ with $\psi \equiv 1$ on $\mathbb{R}^{\leq 0}$ and $\psi \equiv 0$ on $\mathbb{R}^{\geq 1}$. Then we set

$$
\psi[\zeta](x):=\psi\left(\zeta^{-1} \cdot \delta_{\langle A\rangle}(x)-1\right) \text { for } x \in H_{i} \backslash \Sigma_{i} \text { and } \zeta \in(0,1) .
$$

Since $\delta_{\langle A\rangle}$ is Lipschitz continuous there exists some constant $c(\psi)>0$ depending only on $\psi$ such that for the distributional derivative

$$
|\nabla \psi[\zeta](x)| \quad \begin{cases}\leq c(\psi) \cdot\langle A\rangle(x), & \text { for } \delta_{\langle A\rangle}(x) \in(\zeta, 2 \cdot \zeta) \\ =0, & \text { otherwise. }\end{cases}
$$

From Eqs. 21 and 22 we see that

$$
\begin{aligned}
\left|\psi[\zeta] \cdot G^{*}\right|_{H_{\langle A\rangle}^{1,2}\left(\mathbb{I}_{2 \zeta} \backslash \mathbb{I}_{\zeta}\right)} & =\int_{\mathbb{I}_{2 \zeta} \backslash \mathbb{I}_{\zeta}}\left|\nabla\left(\psi[\zeta] \cdot G^{*}\right)\right|^{2}+\langle A\rangle^{2} \cdot\left(\psi[\zeta] \cdot G^{*}\right)^{2} d V \\
& \leq \int_{\mathbb{I}_{2 \zeta} \backslash \mathbb{I}_{\zeta}} 2|\nabla \psi[\zeta]|^{2} \cdot\left(G^{*}\right)^{2}+2\left|\nabla G^{*}\right|^{2}+2\langle A\rangle^{2} \cdot\left(G^{*}\right)^{2} d V \\
& \leq \int_{\mathbb{I}_{2 \zeta} \backslash \mathbb{I}_{\zeta}} 4 c(\psi)^{2} \cdot\langle A\rangle^{2} \cdot\left(G^{*}\right)^{2}+2\left|\nabla G^{*}\right|^{2}+2\langle A\rangle^{2} \cdot\left(G^{*}\right)^{2} d V \\
& \leq 4\left(c(\psi)^{2}+1\right) \cdot\left|G^{*}\right|_{H_{\langle A\rangle}^{1,2}\left(\mathbb{I}_{2 \cdot \zeta} \backslash \mathbb{I}_{\zeta}\right)}
\end{aligned}
$$

Secondly, we consider a cut-off towards infinity by setting $\psi_{R}(x):=\psi\left(R^{-1} \cdot|x|-1\right)$ for $x \in H_{\infty} \backslash \Sigma_{\infty}$ and $R \geq 10$. We note as a counterpart of Eq. 22 that

$$
\left|\nabla \psi_{R}(x)\right| \quad \begin{cases}\leq c(\psi)^{*} / R, & \text { for }|x| \in(R, 2 \cdot R) ; \\ =0, & \text { otherwise }\end{cases}
$$

for some $c(\psi)^{*}>0$. The Lipschitz condition $\left|\delta_{\langle A\rangle_{H_{\infty}}}(x)-\delta_{\langle A\rangle_{H_{\infty}}}\left(a_{\infty}\right)\right| \leq L_{\langle A\rangle}$. $d_{H_{\infty}}\left(x, a_{\infty}\right)$ shows that $\delta_{\langle A\rangle_{H_{\infty}}}(x) \leq L_{\langle A\rangle} \cdot d_{H_{\infty}}\left(x, a_{\infty}\right)+\delta_{\langle A\rangle_{H_{\infty}}}\left(a_{\infty}\right)$. This means that

$$
\langle A\rangle(x) \geq b_{H_{\infty}} \cdot d_{H_{\infty}}\left(x, a_{\infty}\right)^{-1} \text { on } H_{\infty} \backslash\left(\Sigma_{\infty} \cup B_{1}\left(a_{\infty}\right)\right),
$$

for some $b_{H_{\infty}}>0$. Then we have using Eqs. 23 and 24:

$$
\begin{aligned}
\mid\left(\left.\psi_{R} \cdot G^{*}\right|_{H_{\langle A\rangle}^{1,2}\left(B_{2} \cdot R \backslash B_{R}\left(a_{\infty}\right)\right)} ^{2}\right. & \int_{B_{2 \cdot R} \backslash B_{R}\left(a_{\infty}\right)}\left|\nabla\left(\psi_{R} \cdot G^{*}\right)\right|^{2}+\langle A\rangle^{2} \cdot\left(\psi_{R} \cdot G^{*}\right)^{2} d V \\
\leq & 2 \int_{B_{2 \cdot R} \backslash B_{R}\left(a_{\infty}\right)} \psi_{R}^{2} \cdot\left|\nabla G^{*}\right|^{2}+\left|\nabla \psi_{R}\right|^{2} \cdot\left(G^{*}\right)^{2}+\langle A\rangle^{2} \cdot \psi_{R}^{2} \cdot\left(G^{*}\right)^{2} d V \\
\leq & 2 \int_{B_{2 \cdot R} \backslash B_{R}\left(a_{\infty}\right)} \psi_{R}^{2} \cdot\left|\nabla G^{*}\right|^{2}+\langle A\rangle^{2} \cdot \psi_{R}^{2} \cdot\left(G^{*}\right)^{2} d V \\
& \quad+2 \int_{\left.B_{2 \cdot R} \backslash B_{R}\left(a_{\infty}\right)\right)}\left(c(\psi)^{*} / R\right)^{2} \cdot\left(G^{*}\right)^{2} d V \\
\leq & 2 \cdot\left(1+\left(c(\psi)^{*} / b_{H_{\infty}}\right)^{2}\right) \cdot\left|G^{*}\right|_{H_{\langle A\rangle}^{1,2}\left(B_{2 \cdot R} \backslash B_{R}\left(a_{\infty}\right)\right)} .
\end{aligned}
$$

We observe, from $G^{*} \in H_{\langle A\rangle}^{1,2}\left(H_{\infty} \backslash\left(U_{\infty} \cup \Sigma_{H_{\infty}}\right)\right)$, that $\left|G^{*}\right|_{H_{\langle A\rangle}^{1,2}\left(\mathbb{I}_{2 \cdot \zeta} \backslash \mathbb{I}_{\zeta}\right)}$ and $\left|G^{*}\right|_{H_{\langle A\rangle}^{1,2}\left(B_{2 \cdot R} \backslash B_{R}\left(a_{\infty}\right)\right)}$ tend to 0 , when $\varepsilon \rightarrow 0$ respectively $R \rightarrow \infty$. Transferring the cut-off region

$$
C[R, \varepsilon]:=\left(B_{2 \cdot R}\left(a_{\infty}\right) \cap \mathbb{I}_{2 \cdot \varepsilon} \backslash \mathbb{I}_{\varepsilon}\right) \cup\left(B_{2 \cdot R} \backslash B_{R}\left(a_{\infty}\right)\right) \backslash \mathbb{I}_{\varepsilon}
$$

via the ID-map to $H_{i}$ shows that for sufficiently small $\varepsilon>0$, large $R$ and $i$, the contributions to the two norms on the cut-off region become arbitrarily small:

$$
\left|\psi[\varepsilon] \cdot G_{i}\right|_{H_{\langle A\rangle}^{1,2}(\mathbf{I D}(C[R, \varepsilon]))} \ll 1 \text { and thus }\left|J_{(\mathbf{I D}(C[R, \varepsilon]))}\left(\psi[\varepsilon] \cdot G_{i}\right)\right| \ll 1
$$


due to the $\langle A\rangle$-adaptedness of $L$. That is, we get for large $i$

$$
\left(1-\psi_{R}\right) \cdot \psi[\varepsilon] \cdot G_{i} \in H_{\langle A\rangle}^{1,2}\left(H_{i} \backslash \Sigma_{H_{i}}\right) \quad \text { and } \quad J_{H_{i}}\left(\left(1-\psi_{R}\right) \cdot \psi[\varepsilon] \cdot G_{i}\right)<-c / 2
$$

But this contradicts the $\mathcal{S}$-adaptedness of $L$ for test functions in $H_{\langle A\rangle}^{1,2}\left(H_{i} \backslash \Sigma_{H_{i}}\right)$ as this asserts that $J_{H_{i}}\left(\left(1-\psi_{R}\right) \cdot \psi[\varepsilon] \cdot G_{i}\right)>0$, cf. Lemma 3.15 .

Remark 3.18 The considered domain of a shifted $\mathcal{S}$-adapted operator $L$ is typically a space of (sufficiently) regular functions. For such domains the operators are symmetric on the original space $H \backslash \Sigma$, but in general not self-adjoint. (Note in passing that the associated operator $\delta_{\langle A\rangle}^{-2} \cdot L$ on the hyperbolic unfolding is usually no longer a symmetric operator.)

However, right from the definition, any shifted $\mathcal{S}$-adapted operator $L$ is a symmetric semi-bounded operator on $C_{0}^{1}(H \backslash \Sigma)$. This is sufficient to ensure that $L$ admits a canonical self-adjoint extension $L_{F}$, the so-called Friedrichs extension. Its domain $D\left(L_{F}\right)$ satisfies

$$
C_{0}^{1}(H \backslash \Sigma) \subset D\left(L_{F}\right) \subset H_{\langle A\rangle}^{1,2}(H \backslash \Sigma) .
$$

For these Friedrichs extensions we have a general existence and uniqueness theory for the Dirichlet problem in Step 2 of Proposition 3.16 above, cf. [15, Ch.4.3] and [24, Ch.5.5].

The boundary Harnack inequality allows us to derive the following localized versions of this stability of minimal Green's functions.

Proposition 3.19 (Localized Inheritance of $L$-Vanishing) We assume situation $S_{1}$ or $S_{2}$. Then, let $u_{i}>0$ be a solution of $L \phi=0$ on each $H_{i}$ which via ID-maps are compactly converging to some entire solution $u_{\infty}>0$ of $L \phi=0$ on $H_{\infty}$. If there is a ball $B$ in $a$ common ambient manifold of the $H_{i}$ and $H_{\infty}$ around a singular point $p \in \Sigma_{H_{\infty}}$ such that $u_{i}$, for any $i$, is L-vanishing along $B \cap \Sigma_{H_{i}}$, then $u_{\infty}$ is also L-vanishing along $B \cap \Sigma_{H_{\infty}}$.

We note from [17, Theorem 3.4] that it suffices when the $u_{i}$ and $u_{\infty}$ are supersolutions on $H_{i}$ and $H_{\infty}$ and solutions on $B \cap H_{i}$.

The proof is done in three steps. In Step 1 we prepare canonical $\Phi_{\delta}$-chains on the $H_{i}$ converging to a canonical $\Phi_{\delta}$-chains on $H_{\infty}$ we use as a neighborhood basis of 0 . Next, in Step 2, we observe that for these $\Phi_{\delta}$-chains on the $H_{i}$ we get common constants in the BHP on all $H_{i}$, cf. [17, Ch.2.1]. In Step 3 we use the stability of minimal Green's functions $G_{i}$ on the $H_{i}$ 3.16. Using Step 2, we see that the minimal Green's function $G_{i}$ upper bounds the $u_{i}$ on $B \cap H_{i}$ by a common multiple of $G_{i}$ and this carries over to the limit. Thus, we infer that $u_{\infty}$ is also $L$-vanishing along $B \cap \Sigma_{H_{\infty}}$.

Remark 3.20 For Step 1 we recall from [17, Ch.3.1] that the BHP for $\mathcal{S}$-adapted operators is a direct consequence of Ancona's BHP from the unfolding correspondence. For converging sequences $H_{i}$ as in situations $S_{1}$ and $S_{2}$ the associated Gromov hyperbolic $\left(H_{i}, d_{\langle A\rangle_{H_{i}}}\right)$ converge compactly to that of the limit space $H_{\infty}$. For the Whitney smoothed $\mathcal{S}$-metrics $d_{\langle A\rangle^{*}}$, used in the unfolding correspondence, any such sequence $\left(H_{i}, d_{\langle A\rangle_{H_{i}}^{*}}\right)$ has subsequences converging to a Whitney smoothing $\left(H_{\infty}, d_{\langle A\rangle_{H_{\infty}}^{*}}\right)$. This readily follows from the proof of [17, Proposition B.3] since the basepoints of the $\mathcal{S}$-adpated covers compactly subconverge. In what follows we therefore assume that the $\left(H_{i}, d_{\langle A\rangle_{H_{i}}^{*}}\right)$ already converge to $\left(H_{\infty}, d_{\langle A\rangle_{H_{\infty}}^{*}}\right)$. 


\section{Proof Proof (of 3.19)}

Step 1 We first recall the construction of canonical $\Phi_{\delta}$-chains from [17, Lemma 2.4]. For a $\delta$-hyperbolic space we consider geodesic arcs $\gamma:(0, c) \rightarrow X$, for $c>10^{3} \cdot \delta$. We choose the track points $x_{k}=\gamma\left(t_{k}\right), k=0, \ldots, m+1$, with $x_{0}=\gamma(0), x_{m+1}=$ $\gamma(c)$, with $d\left(x_{k}, x_{k+1}\right)=300 \cdot \delta$, for $k<m$, and $d\left(x_{m}, x_{m+1}\right) \leq 300 \cdot \delta$. Then the $\mathcal{N}_{k}^{\delta}(\gamma):=U_{t_{k}}$ with

$$
U_{t}^{\gamma}:=\{x \in X \mid \operatorname{dist}(x, \gamma([t, c)))<\operatorname{dist}(x, \gamma((0, t]))\} .
$$

form a canonical $\Phi_{\delta}$-chain with track points $x_{k}, k=1, \ldots, m$, for $\Phi_{\delta}(t)=a_{\delta}+b_{\delta} \cdot t$ for some $a_{\delta}, b_{\delta}>0$ depending only on $\delta$. We call $m$ its length.

In both situations $S_{1}$ and $S_{2}$ we now turn to the Whitney smoothed hyperbolic unfoldings. We can choose hyperbolic geodesic arcs $\gamma\left[c_{i}\right]:\left(0, c_{i}\right) \rightarrow H_{i} \backslash \Sigma_{H_{i}}$, with basepoint $\gamma\left[c_{i}\right](0)=c_{i}$ and $c_{i} \rightarrow \infty$ for $i \rightarrow \infty$, so that the $\gamma\left[c_{i}\right]$ compactly converge to some $\gamma\left[c_{\infty}\right] \subset\left(H_{\infty}, d_{\langle A\rangle_{H_{\infty}}^{*}}\right)$ representing the Gromov boundary point $0 \in \Sigma_{H_{\infty}}$. The convergence is formalized via ID-maps.

This also implies that the canonical $\Phi_{\delta}$-chains $\mathcal{N}_{k}^{\delta}\left(\gamma\left[a_{i}\right]\right) \subset H_{i}$, of length $m(i) \rightarrow$ $\infty$, we assign to each of the $\gamma\left[a_{i}\right]$, compactly converge to $\mathcal{N}_{k}^{\delta}\left(\gamma\left[a_{\infty}\right]\right) \subset H_{\infty}$, via ID-maps.

Step 2 We can now apply the BHPs [17, Theorem 3.4 and 3.5]. For a minimal Green's function $G$ and solutions $v>0$ of $L \phi=0$ on $H_{i} \backslash \Sigma_{H_{i}}$ which is $L$-vanishing along $\mathcal{N}_{2}^{\delta}\left(\gamma\left[a_{i}\right]\right)$ we have:

$G(x, p) / v(x) \leq C \cdot G(y, p) / v(y)$, for any $p \in\left(H_{i} \backslash \Sigma_{H_{i}}\right) \backslash \mathcal{N}_{1}^{\delta}\left(\gamma\left[a_{i}\right]\right), x, y \in \mathcal{N}_{3}^{\delta}\left(\gamma\left[a_{i}\right]\right)$.

For $k_{L} \leq \kappa$ and $\varepsilon_{L} \geq \eta$ the constant $C$ only depends on $\kappa$ and $\eta>0$. In our cases this means that we can choose a common Harnack constant $C$ for all $i$. For $S_{1}$ this from the stable BHP on $\mathcal{H}_{n}^{\mathbb{R}}$ [17, Theorem 3.5]. It gives the same constant for all such hypersurfaces. For $S_{2}$ we can employ the BHP on $\mathcal{G}_{n}$ of [17, Theorem 3.4] since we are working only with one fixed hypersurface.

Step 3 In both situations, $S_{1}$ or $S_{2}$, we have $u_{i} \leq C \cdot G_{i}$ on $\mathcal{N}_{3}^{\delta}\left(\gamma\left[a_{i}\right]\right)$ for some common $C \geq 1$. Now we recall from [6, Proposition 8.10] that the canonical $\Phi_{\delta}$-chain $\mathcal{N}_{k}^{\delta}\left(\gamma\left[a_{\infty}\right]\right) \subset H_{\infty}$ for $\gamma\left[a_{\infty}\right]$ forms a neighborhood basis of $0 \in H_{\infty}$. Since $\Sigma_{H_{\infty}} \cap \bar{B}$ is compact, we can therefore assume (without loss of generality) that $B \cap H_{\infty} \subset \mathcal{N}_{k}^{\delta}\left(\gamma\left[a_{\infty}\right]\right)$, for $k=1, \ldots 3, i \geq 1$ and $i=\infty$.

Thus we also have

$$
u_{\infty} \leq C \cdot G_{\infty} \text { on } B \cap H_{\infty} \backslash \Sigma_{H_{\infty}}
$$

Since $G_{\infty}$ is minimal we see that $u_{\infty}$ also $L$-vanishes along $B \cap \Sigma_{H_{\infty}}$.

This result also concludes the proof of 3.12 and 3.13 taking increasingly larger balls $B$ in 3.19 .

\section{Martin Theory on Minimal Cones}

Here we refine our analysis of natural Schrödinger operators. The minimal growth stability and Martin theory can be used to build an inductive asymptotic analysis of solutions with minimal growth near singular points using iterative blow-ups. In the cases of the Jacobi field operator and the conformal Laplacian we derive some further details. 


\subsection{Schrödinger Operators and Scaling Actions}

For Schrödinger operators $L$ which are naturally associated with $H \in \mathcal{G}$ we get a neat representation for certain distinguished solutions of $L \phi=0$. We estimate their radial growth in the cone case to gain a detailed understanding of the growth of the Martin kernel for compact $H$.

Definition 4.1 (Natural Schrödinger Operators) A natural and shifted $\mathcal{S}$-adapted operator $L$ is called a natural Schrödinger operator if for any given $H \in \mathcal{H}$ the operator $L(H)$ has the form

$$
L(H)(u)=-\Delta_{H} u+V_{H}(x) \cdot u \text { on } H \backslash \Sigma_{H}
$$

for some Hölder continuous function $V_{H}(x)$.

Remark 4.2 (Inheritance versus Naturality) Note that a natural Schrödinger operator is merely shifted $\mathcal{S}$-adapted with respect to a given $H \in \mathcal{H}$. However, due to Inheritance Lemma 3.9, naturality always implies that for a blow-up $N$ of $H$ around a singular point in $\Sigma_{H}, L(N)$ is shifted $\mathcal{S}$-adapted with $\lambda_{L, H}^{\langle A\rangle} \leq \lambda_{L, N}^{\langle A\rangle}$.

This class of Schrödinger operators covers the examples we discussed in Chapter 2.4, namely the Laplacian $-\Delta_{H}$, the Jacobi field operator $J_{H}=-\Delta_{H}-|A|^{2}-R i c_{M}(v, v)$ and the $S$-conformal Laplacian $L_{H, S}=-\Delta_{H}+\frac{n-2}{4(n-1)} \cdot\left(\operatorname{scal}_{H}-\left.S\right|_{H}\right)$.

For an area minimizing cone $C \subset \mathcal{H}$ the naturality implies that $V_{C}(t \cdot x)=t^{-2} \cdot V_{C}(x)$ for any $x \in C \backslash \sigma_{C}$ and $t>0$. In this case we can write $V(x)=r^{-2} \cdot V^{\times}(\omega)$ for $x=$ $(\omega, r) \in S_{C} \backslash \sigma_{C} \times \mathbb{R}^{>0}=C \backslash \sigma_{C}$. It follows that

$L v=-\frac{\partial^{2} v}{\partial r^{2}}-\frac{n-1}{r} \cdot \frac{\partial v}{\partial r}-\frac{1}{r^{2}} \cdot \Delta_{S_{C}} v+\frac{1}{r^{2}} \cdot V^{\times}(\omega) \cdot v=:-\frac{\partial^{2} v}{\partial r^{2}}-\frac{n-1}{r} \cdot \frac{\partial v}{\partial r}+\frac{1}{r^{2}} \cdot L^{\times} v$,

where $L^{\times}=L^{\times}\left(S_{C}\right)$ is an operator on $S_{C}$. We will see in 4.4 below that $L^{\times}$is also a natural Schrödinger operator.

Scaling Actions On an area minimizing cones equipped with a natural and $\mathcal{S}$-adapted operator $L$, both, the operator and solutions of $L \phi=0$ reproduce themselves under scalings of the cone up to constant multiples. Concretely, we express $L$ on $C=\mathbb{R}^{\geq 0} \times S_{C}$ in geodesic coordinates $x_{1}=r, x_{2}, \ldots, x_{n}$ such that $x_{2}, \ldots, x_{n}$ locally parametrize $S_{C}=C \cap \partial B_{1}(0)$ :

$$
-L(u)=\sum_{i, j} a_{i j} \cdot \frac{\partial^{2} u}{\partial s_{i} \partial s_{j}}+\sum_{i} b_{i} \cdot \frac{\partial u}{\partial s_{i}}+c \cdot u .
$$

When $L$ is natural this means that for any $\eta>0$,

$$
a_{i j}(\eta \cdot x)=a_{i j}(x), b_{i}(\eta \cdot x)=\eta^{-1} \cdot b_{i}(x) \text { and } c(\eta \cdot x)=\eta^{-2} \cdot c(x) .
$$

Thus, for any function $u(x)$ that solves $L \phi=0$ its rescaling $u(\eta \cdot x)$ also solves this equation by the chain rule. In particular, the Green's function and the set of minimal solutions of $L \phi=0$ are reproduced up to multiples under composition with the scaling map

$$
S_{\eta}: C \rightarrow C \text { given by } x \mapsto \eta \cdot x \text { for } \eta \in(0, \infty) .
$$


More concretely, we consider the map $u \mapsto u \circ S_{\eta}$ and normalize the values of the resulting functions $u \circ S_{\eta}$ to 1 in some base point $p \in C \backslash \sigma_{C}$. In this way we define a scaling action $S_{\eta}^{*}$ on the Martin boundary.

Lemma 4.3 (Attractors and Fixed Points of $\mathbf{S}_{\eta}^{*}$ ) Let $C \in \mathcal{S C}_{n}$ be a singular area minimizing cone and $L$ be a natural $\mathcal{S}$-adapted operator on $C$. Then we have:

(i) The scaling action $S_{\eta}^{*}$ on $\partial_{M}(C, L)$ has exactly two fixed points: the tip $0_{C}$ and the point at infinity $\infty_{C}$, both viewed as minimal functions.

(ii) For $z \in \partial_{M}(C, L) \backslash\left\{0_{C}, \infty_{C}\right\}$ we find

$$
S_{\eta}^{*}(z) \rightarrow \infty \text { as } \eta \rightarrow \infty \text { and } S_{\eta}^{*}(z) \rightarrow 0_{C} \text { as } \eta \rightarrow 0 .
$$

(iii) More generally, for any given $z \in C \backslash \sigma_{C}$ we have

$$
\frac{G\left(x, S_{\eta}(z)\right)}{G\left(p, S_{\eta}(z)\right)} \rightarrow \infty_{C} \text { as } \eta \rightarrow \infty \text { and } \frac{G\left(x, S_{\eta}(z)\right)}{G\left(p, S_{\eta}(z)\right)} \rightarrow 0_{C} \text { as } \eta \rightarrow 0
$$

locally uniformly for $x \in C \backslash \sigma_{C}$.

Proof This readily follows from the way the pole of $G\left(\cdot, S_{\eta}(z)\right)$ shifts under these scaling operations.

Now we give a description of the two fixed point solutions in $\partial_{M}(C, L)$. Despite the fact mentioned above that there is no proper way of transforming the Martin theory from the original hypersurface $H$ to its tangent cones this will allow us to build an inductive decomposition scheme for solutions on $H$.

Theorem 4.4 (Separation of Variables) For any cone $C \in \mathcal{S C}_{n}$ and any natural Schrödinger operator $L$ let us consider the $\mathcal{S}$-adapted operator $L_{\lambda}=L-\lambda \cdot\langle A\rangle^{2} \cdot I d$, $\lambda<\lambda_{L, C}^{\langle A\rangle}$. Then we have:

- Viewed as functions $\Psi_{-}=0_{C}$ and $\Psi_{+}=\infty_{C}$ on $C \backslash \sigma$ the two fixed points $0_{C}$, $\infty_{C} \in \partial_{M}\left(C, L_{\lambda}\right)$ can be written as

$$
\Psi_{ \pm}(\omega, r)=\psi(\omega) \cdot r^{\alpha_{ \pm}}
$$

with $(\omega, r) \in S_{C} \backslash \sigma \times \mathbb{R}$ and $\alpha_{ \pm}=-\frac{n-2}{2} \pm \sqrt{\left(\frac{n-2}{2}\right)^{2}+\mu_{C, L_{\lambda}^{\times}}}$

- Let $\langle A\rangle^{\times}(\omega)=r \cdot\langle A\rangle(x)$ for $x=(\omega, r) \in C \backslash \sigma$. The associated operator

$$
L_{\lambda}^{\times}(v)(\omega)=-\Delta_{S_{C}} v(\omega)+\left(V^{\times}(\omega)-\lambda \cdot\langle A\rangle^{\times}(\omega)^{2}\right) \cdot v(\omega)
$$

defined on $S_{C} \backslash \Sigma_{S_{C}}$ is a natural Schrödinger operator with non-weighted principal eigenvalue $\mu_{C, L_{\lambda}^{\times}}>-\left(\frac{n-2}{2}\right)^{2}$ and ground state $\psi(\omega)>0$, that is,

$$
L_{\lambda}^{\times} \psi=\mu_{C, L_{\lambda}^{\times}} \cdot \psi \text {, on } S_{C} \backslash \Sigma_{S_{C}} .
$$

Proof We proceed in three steps: First, we show that $\Psi_{ \pm}$can be written as a product $\Psi_{ \pm}(\omega, r)=\psi_{ \pm}(\omega) \cdot r^{\alpha_{ \pm}}$. Then we determine $\psi_{ \pm}$and $\alpha_{ \pm}$for some inner approximation $C$ by regular subcones. Finally, we prove that the resulting values converge to $\psi_{ \pm}$and $\alpha_{ \pm}$on $C$. 
Product Shape We first restrict the two fixed points $\Psi_{ \pm} \in \partial_{M}\left(C, L_{\lambda}\right)$ to a regular ray $\Gamma_{v}=\mathbb{R}^{>0} \cdot v \in C \backslash \sigma$, for some $v \in S_{C}$. Consider the map $\left.\Psi_{ \pm}\right|_{\Gamma_{v}}: \mathbb{R}^{>0} \cdot v \rightarrow \mathbb{R}^{>0}$ which we view as a restriction of a function $f: \mathbb{R}^{>0} \rightarrow \mathbb{R}^{>0}$. Now up to a constant $\Psi_{ \pm}$ reproduces under scalings: For any $\eta>0$, there is constant $c_{\eta}>0$ such that

$$
f(\eta \cdot x)=c_{\eta} \cdot f(x) \text { for all } x \in \mathbb{R}^{>0} .
$$

From this it follows that $f$ is a monomial, that is, $f(x)=a \cdot x^{b}$ for some constants $a(v)>0$, $b(v) \in \mathbb{R}$. This argument applies to any regular ray $\Gamma_{v}$.

Next we consider the Harnack inequality for $L_{\lambda}$ on a ball $B_{2 R}(v) \subset C \backslash \sigma_{C}$ for some $R>0$. We get, for any positive solution $u$ of $L_{\lambda} \phi=0$, the Harnack inequality

$$
\sup _{B_{R}(v)} u \leq c\left(L_{\lambda}, v, R\right) \cdot \inf _{B_{R}(v)} u,
$$

for some constant independent of $u$. The crux of the matter is that both the scaling symmetry of $C$ and the naturality of $L_{\lambda}$ imply that the same constant $c$ can still be used in the Harnack inequality after scalings around the tip 0: For any $s_{0}$ we have

$$
\sup _{B_{s \cdot R}(s \cdot v)} u \leq c\left(L_{\lambda}, v, R\right) \cdot \inf _{B_{s \cdot R}(s \cdot v)} u .
$$

This implies that for all rays $\Gamma_{w}$ passing through $B_{R}(v)$ the exponent $b(w)$ equals $b(v)$. Since $S_{C} \backslash \Sigma_{S_{C}}$ is connected, this shows that $b(v)$ is constant on $S_{C} \backslash \Sigma_{S_{C}}$. Thus $\Psi_{ \pm}$can be written as $\Psi_{ \pm}(\omega, r)=\psi_{ \pm}(\omega) \cdot r^{\alpha_{ \pm}}$for $(\omega, r) \in \partial B_{1}(0) \cap C \backslash \sigma \times \mathbb{R}^{>0}$ and some $C^{2, \beta}$-regular function $\psi_{ \pm}$on $\partial B_{1}(0) \cap C \backslash \sigma_{C} \times \mathbb{R}^{>0}, \beta>0$.

We reinsert $\Psi_{ \pm}(\omega, r)=\psi_{ \pm}(\omega) \cdot r^{\alpha_{ \pm}}$into the equation $L_{\lambda} \phi=0$ which we write in polar coordinates as in Eq. 28. A separation of variables shows that the $\psi_{ \pm}$solve the equations

$$
L_{\lambda}^{\times} v:=-\Delta_{S_{C}} v(\omega)+\left(V^{\times}(\omega)-\lambda \cdot\left(\langle A\rangle^{\times}\right)^{2}(\omega)\right) \cdot v(\omega)=\left(\alpha_{ \pm}^{2}+(n-2) \cdot \alpha_{ \pm}\right) \cdot v
$$

on $S_{C} \backslash \Sigma_{S_{C}}$. Further, $L_{\lambda}^{\times}$is again a natural Schrödinger operator and adapted to the $\mathcal{S}$ transform $\langle A\rangle^{\times}=\left.\langle A\rangle_{C}\right|_{S_{C}}$.

Inner Regular Approximation We use an approximation by Dirichlet eigenvalue problems to show that

- $\alpha_{-}^{2}+(n-2) \cdot \alpha_{-}=\alpha_{+}^{2}+(n-2) \cdot \alpha_{+}>-(n-2)^{2} / 4$.

- $\mu_{C, L_{\lambda}^{\times}}:=\alpha_{ \pm}^{2}+(n-2) \cdot \alpha_{ \pm}$is the non-weighted principal eigenvalue of $L_{\lambda}^{\times}$. Further, $L_{\lambda}^{\times}$is shifted $\mathcal{S}$-adapted.

- $\quad \psi_{-}=\psi_{+}$and $\psi:=\psi_{ \pm}$is the corresponding ground state of $L_{\lambda}^{\times}$.

Towards this end we choose smoothly bounded domains $D_{i} \subset \bar{D}_{i} \subset S_{C} \backslash \Sigma_{S_{C}}$ with $D_{i} \subset$ $D_{i+1}$ and $\bigcup_{i} D_{i}=S_{C} \backslash \Sigma_{S_{C}}$. We consider the positive solutions of $L_{\lambda} \phi=0$ on the cone $C\left(D_{i}\right) \subset C$ over $D_{i}$ with vanishing boundary value along $\partial C\left(D_{i}\right) \backslash\{0\}$.

Now we apply [17, Remark 3.10] to $L_{\lambda}$ on $C\left(D_{i}\right)$. Note that there exists $a_{C\left(D_{i}\right)}>0$ such that $\operatorname{dist}\left(z, \Sigma_{S_{C}}\right) \leq a_{C\left(D_{i}\right)} \cdot \delta_{\langle A\rangle}(z) \leq a_{C\left(D_{i}\right)} \cdot L_{\langle A\rangle} \cdot \operatorname{dist}\left(z, \Sigma_{, a S_{C}}\right)$. One readily checks the adaptedness conditions. Therefore, the Martin boundary of $L_{\lambda}$ equals $\left(\partial C\left(D_{i}\right) \backslash\right.$ $\{0\}) \cup\left\{0_{C\left(D_{i}\right)}, \infty_{C\left(D_{i}\right)}\right\}$ and the two fixed point solutions $\Psi_{ \pm}[i]$ of $L_{\lambda} v$ vanishing along $\partial C\left(D_{i}\right) \backslash\{0\}$, alternatively labelled $0_{C\left(D_{i}\right)}, \infty_{C\left(D_{i}\right)}$, are again of the form $\Psi_{ \pm}[i](\omega, r)=$ $\psi_{ \pm}[i](\omega) \cdot r^{\alpha[i]_{ \pm}}$.

Again, we insert $\Psi_{ \pm}[i](\omega, r)$ into the equation $L_{\lambda} \phi=0$, written in polar coordinates as in Eq. 28 and find that the $\psi_{ \pm}[i]$ solve

$$
L_{\lambda}^{\times} v=-\Delta_{S_{C}} v(\omega)+\left(V^{\times}(\omega)-\lambda \cdot\left(\langle A\rangle^{\times}\right)^{2}(\omega)\right) \cdot v(\omega)=\left(\alpha_{ \pm}[i]^{2}+(n-2) \cdot \alpha_{ \pm}[i]\right) \cdot v .
$$


Over $D_{i}$ we apply the spectral theory for bounded domains and observe that the positive eigenfunctions $\psi_{ \pm}[i]$ must equal the uniquely determined first Dirichlet eigenfunction $\psi[i]$ for the first eigenvalue $\mu[i]$ of $L_{\lambda}^{\times}$. Thus we have

$$
\psi[i]=\psi_{-}[i]=\psi_{+}[i] \text { and } \mu[i]=\alpha_{-}[i]^{2}+(n-2) \cdot \alpha_{-}[i]=\alpha_{+}[i]^{2}+(n-2) \cdot \alpha_{+}[i],
$$

whence $\mu[i] \geq-(n-2)^{2} / 4$. Further, the variational characterization of these eigenvalues gives $\mu[i] \geq \mu[i+1]$, since the space of admissible test functions on $D_{i}$ is a subset of the corresponding function space over $D_{i+1}$.

After a suitable normalization the first Dirichlet eigenfunctions $\psi[i]$ of $L_{\lambda}^{\times}$on $D_{i}$ converge $C^{3}$-compactly on $S_{C} \backslash \Sigma_{S_{C}}$ as $i \rightarrow \infty$ to a positive eigenfunction $\psi^{*}$ with eigenvalue $\mu^{*}=\lim _{i \rightarrow \infty} \mu[i] \geq-(n-2)^{2} / 4$. We also have the limits $\alpha_{ \pm}^{*}:=\lim _{i \rightarrow \infty} \alpha_{ \pm}[i]$. From this we observe as in [17, Theorem 5.7] that $\psi^{*}>0$ is the non-weighted ground state of $L_{\lambda}^{\times}$on $S_{C} \backslash \Sigma_{S_{C}}$ for the eigenvalue $\mu^{*}>-\infty$. Moreover, $L_{\lambda}^{\times}$, and hence $L^{\times}$, are shifted $\mathcal{S}$ adapted. Namely, $\langle A\rangle^{\times}>c_{S_{C}}>0$ and thus the principal eigenvalue of $\delta_{\langle A\rangle^{\times}}^{2} \cdot L_{\lambda}^{\times}$remains finite as the non-weighted principal eigenvalue $\mu^{*}$ of $L_{\lambda}^{\times}$is finite. Therefore, $L_{\lambda}^{\times}$is a natural Schrödinger operator.

Comparison Arguments Now we compare these solutions with $0_{C}, \infty_{C} \in \partial_{M}\left(C, L_{\lambda}\right)$. From the fact that there are no positive solutions for $L_{\lambda}^{\times} v=\mu \cdot v$ we deduce that if $\mu<\mu^{*}$,

$$
\alpha_{ \pm}^{2}+(n-2) \cdot \alpha_{ \pm} \geq \mu^{*} \geq-(n-2)^{2} / 4 .
$$

In turn, the solution $\Psi_{+}=\psi(\omega) \cdot r^{\alpha_{+}} L$-vanishes along $\widehat{\sigma_{C}} \backslash\{\infty\}$ and in particular in 0 . This implies the estimate

$$
\alpha_{+}^{*} \geq \alpha_{+} \text {and therefore } \mu^{*} \geq \alpha_{ \pm}^{2}+(n-2) \cdot \alpha_{ \pm} .
$$

From Eqs. 32 and 33 we conclude

$$
\mu^{*}=\alpha_{ \pm}^{2}+(n-2) \cdot \alpha_{ \pm} \text {and therefore } \alpha_{ \pm}=\alpha_{ \pm}^{*} .
$$

Since $0_{C} \neq \infty_{C} \in \partial_{M}\left(C, L_{\lambda}\right)$, we further infer that

$$
\alpha_{-}<-(n-2) / 2<\alpha_{+} \text {and } \alpha_{ \pm}^{2}+(n-2) \cdot \alpha_{ \pm}>-(n-2)^{2} / 4 .
$$

Finally, the functions $\psi_{ \pm}$belong to the eigenvalue $\alpha_{ \pm}^{2}+(n-2) \cdot \alpha_{ \pm}=\mu^{*}$. Since the ground state is uniquely determined, $\psi:=\psi_{-}=\psi_{+}=\psi^{*}$ is the ground state of $L_{\lambda}^{\times}$.

\subsection{Geometric Operators on Cones}

Next we focus on a subclass of natural Schrödinger operators which one typically encounters in applications to scalar curvature geometry. For these we will derive quite explicit growth estimates.

Theorem 4.5 (Eigenvalue Estimates for $J_{C}$ and $L_{C}$ ) Let $C \in \mathcal{S C}_{n}$ be a singular area minimizing cone. Then we get the following estimates for the Jacobi field operator $J_{C}$ and the conformal Laplacian $L_{C}$ :

- $\quad$ The principal eigenvalue $\lambda_{J_{C}, C}$ of $J_{C}$ is non-negative and

$$
\mu_{C,\left(J_{C}\right)_{\lambda}^{\times}} \geq-\left(\frac{n-2}{2}\right)^{2} \text { for any } \lambda \leq \lambda_{J_{C}, C} .
$$


- $\quad$ There are constants $\Lambda_{n}>\lambda_{n}>0$ depending only on $n$ such that $\left(L_{C}\right)_{\lambda}$ is $\mathcal{S}$-adapted for any $\lambda \leq \Lambda_{n}$. Furthermore,

$$
\mu_{C,\left(L_{C}\right)_{\lambda}^{\times}} \geq-1 / 4 \cdot\left(\frac{n-2}{2}\right)^{2} \text { for any } \lambda \leq \lambda_{n}
$$

and thus

$$
\alpha_{+} \geq-(1-\sqrt{3 / 4}) \cdot \frac{n-2}{2}, \quad \alpha_{-} \leq-(1+\sqrt{3 / 4}) \cdot \frac{n-2}{2} \text { for } \lambda \leq \lambda_{n} .
$$

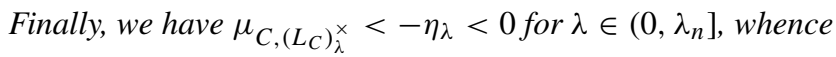

$$
-\vartheta_{\lambda}>\alpha_{+} \geq-(1-\sqrt{3 / 4}) \cdot \frac{n-2}{2}>-\frac{n-2}{2}>-(1+\sqrt{3 / 4}) \cdot \frac{n-2}{2} \geq \alpha_{-}>\vartheta_{\lambda}-(n-2)
$$

for constants $\eta_{\lambda}$ and $\vartheta_{\lambda}>0$ depending only on $\lambda$ and $n$.

Proof From Proposition 2.8 (iv) we know that $\lambda_{J_{C}, C} \geq 0$. For $\lambda<\lambda_{J_{C}, C}, J_{C}$ is $\mathcal{S}$-adapted and thus we have the lower bound $\mu_{C,\left(J_{C}\right)_{\lambda}^{\times}}>-(n-2)^{2} / 4$. Moreover, for any given $\varepsilon>0$, we find from Theorem 4.4 a common upper bound for $\mu_{C,\left(J_{C}\right)_{\lambda}^{\times}}$for all $\lambda$ with $\lambda<\lambda_{J_{C}, C} \leq \lambda+\varepsilon$.

Next we recall that the ground state $v>0$ is uniquely determined. By standard elliptic theory applied to the given set of eigenvalue equations, $v$ is the unique $C^{3}$-limit of solutions $v_{n}>0$ of the shifted operator $\left(J_{C}\right)_{\lambda_{J_{C}, C}-1 / n} \phi=0, n \geq 1$. Note that in this case we have locally uniform bounds on the coefficients. Hence $\mu_{C,\left(J_{C}\right)_{\lambda_{J}, C}^{\times}} \geq-(n-2)^{2} / 4$.

For $L_{C}$ we first notice that there is a common $\Lambda_{n}>0$ for all area minimizing cones in $\mathbb{R}^{n+1}$ such that $\left(L_{C}\right)_{\lambda}$ is $\mathcal{S}$-adapted for all $\lambda \leq \Lambda_{n}$ : For an individual cone this is just Proposition 2.8 (i). The uniform estimate follows from the compactness of the space of singular cones. To proceed further, we note

- the estimate $\mu_{C,\left(J_{C}\right)_{0}^{\times}} \geq-\left(\frac{n-2}{2}\right)^{2}$.

- the Hardy inequality for the $\mathcal{S}$-transform $\left.\langle A\rangle\right|_{S_{C}}$; we can choose the constant $\tau(\langle A\rangle, C)=\tau_{n}>0$ uniformly for all cones. This follows from the naturality of $\langle A\rangle$.

- the identity $\operatorname{scal}_{C} \equiv-|A|^{2}$ which is valid for minimal hypersurfaces in Ricci flat space, cf. the Gauß-Codazzi Eq. 6.

This allows us to estimate the variational integral for $\mu_{C,\left(L_{C}\right)_{\lambda}^{\times}}$for any smooth function $f$ compactly supported in $S_{C} \backslash \Sigma_{S_{C}}=\partial B_{1}(0) \cap C \backslash \sigma_{C}$ :

$$
\begin{aligned}
& \int_{S_{C}} f \cdot\left(L_{C}\right)_{\lambda}^{\times} f d V \\
= & \int_{S_{C}}|\nabla f|^{2}+\left(\left.\frac{n-2}{4(n-1)} \cdot \operatorname{scal}_{C}\right|_{S_{C}}-\left.\lambda \cdot\langle A\rangle\right|_{S_{C}} ^{2}\right) \cdot f^{2} d V \\
\geq & \int_{S_{C}}|\nabla f|^{2}-\frac{n-2}{4(n-1)} \cdot|A|^{2} \cdot f^{2} d V-\max \left\{0, \lambda / \tau_{n}\right\} \cdot \int_{S_{C}}|\nabla f|^{2}+|A|^{2} \cdot f^{2} d V .
\end{aligned}
$$

For $\lambda / \tau_{n}<\frac{1}{4(n-1)}$ and $|f|_{L^{2}}=1$ this yields the lower bound

$$
\geq \frac{1}{4} \int_{S_{C}}|\nabla f|^{2}-|A|^{2} \cdot f^{2} d V \geq \frac{1}{4} \cdot \mu_{C,\left(J_{C}\right)_{0}^{\times}} \geq-\frac{1}{4}\left(\frac{n-2}{2}\right)^{2} .
$$


Hence for $\lambda_{n}:=\min \left\{\Lambda_{n}, \frac{\tau_{n}}{4(n-1)}\right\} / 2$ we find

$$
\mu_{C,\left(L_{C}\right)_{\lambda}^{\times}} \geq-\frac{1}{4}\left(\frac{n-2}{2}\right)^{2} \text { for } \lambda \leq \lambda_{n} .
$$

Next let $\lambda \in\left(0, \lambda_{n}\right]$. Since $s c a l_{C} \leq 0$ and the codimension of $\Sigma_{S_{C}}$ is greater than two, we can find a function $f \in C_{0}^{1}\left(S_{C} \backslash \Sigma_{S_{C}}\right)$ equal to 1 outside a sufficiently small neighborhood of $\Sigma_{S_{C}}$, and equal to 0 close to $\Sigma_{S_{C}}$ so that

$$
\int_{S_{C}}|\nabla f|^{2}+\left(\left.\frac{n-2}{4(n-1)} \cdot \operatorname{scal}_{C}\right|_{S_{C}}-\left.\lambda \cdot\langle A\rangle\right|_{S_{C}} ^{2}\right) \cdot f^{2} d V<-\left.\frac{1}{2} \int_{S_{C}} \lambda \cdot\langle A\rangle\right|_{S_{C}} ^{2} \cdot f^{2} d V<0 .
$$

Due to

- the naturality of $\mathcal{S}$-transforms, and

- the compactness of the space of all singular area minimizing cones $C \subset \mathbb{R}^{n+1}$ in flat norm topology and in compact $C^{5}$-topology outside the singular sets,

we know that there is a common positive lower bound on $\left.\int_{S_{C}}\langle A\rangle\right|_{S_{C}} ^{2} d V$ for all such $C$. More precisely, there are constants $\eta_{\lambda}, \vartheta_{\lambda}>0$ which depend only on $\lambda>0$ and $n$ such that $\mu_{C,\left(L_{C}\right)_{\lambda}^{\times}}<-\eta_{\lambda}<0$, whence

$$
0>-\vartheta_{\lambda}>\alpha_{+} \text {and } \alpha_{-}>\vartheta_{\lambda}-(n-2)>-(n-2)
$$

for any singular area minimizing cone $C$.

Inductive cone reduction arguments are a powerful tool to reduce general questions to low dimensions. In essence, this is an iterative blow-up process where we first blow-up around some $p_{0} \in \Sigma_{H}$ and get a tangent cone $C_{1}$. While scaling around $0 \in \sigma_{C_{1}}$ merely reproduces $C_{1}$, blowing up around a singular point $p_{1} \neq 0 \in \sigma_{C_{1}}$ generates an area minimizing cone $C_{2}$ which can be written as a Riemannian product $C_{2}=\mathbb{R} \times C_{3}$ for a lower dimensional area minimizing cone $C_{3}$. This way we encounter cones $C^{n} \subset \mathbb{R}^{n+1}$ which can be written as a Riemannian product $\mathbb{R}^{n-k} \times C^{k}$, where $C^{k} \subset \mathbb{R}^{k+1}$ is a lower dimensional area minimizing cone singular at 0 . A famous instance of this technique is Federer's estimate for the codimension of the singularity set.

For applications to scalar curvature geometry which we shall consider elsewhere, it is useful to establish this kind of cone reduction arguments for the conformal Laplacian. In this case we observe that the minimal function $\Psi_{+}(\omega, r)$ of $\left(L_{C^{n}}\right)_{\lambda}$ on $C^{n}$ shares the $\mathbb{R}^{n-k_{-}}$ translation symmetry with the underlying space $\mathbb{R}^{n-k} \times C^{k}$ since $\Psi_{+}(\omega, r)$ is uniquely determined. Hence $\left.\Psi_{+}(\omega, r)\right|_{\{0\} \times C^{k}}$ satisfies the equation

$$
\left.\left(L_{C^{k}, n}\right)_{\lambda} \Psi_{+}(\omega, r)\right|_{\{0\} \times C^{k}}=0,
$$

where

$$
L_{C^{k}, n}:=-\Delta-\frac{n-2}{4(n-1)} \cdot|A|^{2} \text { for } n \geq k \text { and }\left(L_{C^{k}, n}\right)_{\lambda}=L_{C^{k}, n}-\lambda \cdot\langle A\rangle^{2} .
$$

Thus $L_{C^{k}, n}$ is a dimensionally shifted version of the conformal Laplacian on the cone $C^{k}$ : The entities $\Delta,|A|^{2}$ and $\langle A\rangle^{2}$ are intrinsically defined on $C^{k}$, while the dimensional shift comes from using $\frac{n-2}{4(n-1)}$ in place of $\frac{k-2}{4(k-1)}$. The next two results describe the analysis of these operators. 
Proposition 4.6 (Dimensionally Shifted $L_{C}$ ) Let $C^{k} \subset \mathbb{R}^{k+1}$ be a singular area minimizing cone and $n \geq k$. Then we have:

- $\quad L_{C, n}$ is $\mathcal{S}$-adapted and for its principal eigenvalue there exists a uniform lower bound for all $k$-dimensional cones by a positive constant $\Lambda_{k}^{*}>0$ independent of $n$.

- $\quad$ There is a constant $\lambda_{k}^{*} \in\left(0, \Lambda_{k}^{*}\right)$ depending only on $k$ such that for any $n \geq k$ and $\lambda \leq \lambda_{k}^{*}$

and thus

$$
\mu_{C,\left(L_{C, n}\right)_{\lambda}^{\times}} \geq-\frac{(k-2)^{2}}{12}
$$

$$
\alpha_{+} \geq-\left(1-\sqrt{\frac{2}{3}}\right) \cdot \frac{k-2}{2}, \quad \alpha_{-} \leq-\left(1+\sqrt{\frac{2}{3}}\right) \cdot \frac{k-2}{2} .
$$

Moreover, we have $\mu_{C,\left(L_{C, n}\right)_{\lambda}^{\times}}^{*}<-\eta_{\lambda}^{*}<0$ for $\lambda \in\left(0, \lambda_{k}^{*}\right]$ and thus

$$
-\vartheta_{\lambda}^{*}>\alpha_{+} \geq-\left(1-\sqrt{\frac{2}{3}}\right) \cdot \frac{k-2}{2}>-\frac{k-2}{2}>-\left(1+\sqrt{\frac{2}{3}}\right) \cdot \frac{k-2}{2} \geq \alpha_{-}>\vartheta_{\lambda}^{*}-(k-2)
$$

for some constants $\eta_{\lambda}^{*}$ and $\vartheta_{\lambda}^{*}>0$ depending only on $\lambda$ and $k$.

Proof From Eq. 8 we get

$$
\begin{aligned}
& \int_{C}|\nabla f|^{2}-\frac{n-2}{4(n-1)} \cdot|A|^{2} \cdot f^{2} d V \quad \geq \int_{C}|\nabla f|^{2}-\frac{1}{4} \cdot|A|^{2} \cdot f^{2} d V \\
& \geq \int_{C} \frac{k}{2(k-1)} \cdot|\nabla f|^{2}+\frac{k-3}{4(k-1)} \cdot|A|^{2} \cdot f^{2} d V,
\end{aligned}
$$

and for some $\tau_{k}^{*}>0$ depending only on the dimension this can be refined to

$$
\geq \frac{k-3}{4(k-1)} \cdot \int_{C} f \cdot C_{H ; A, 0} f d V \geq \tau_{k}^{*} \cdot \frac{k-3}{4(k-1)} \cdot \int_{C}\langle A\rangle^{2} \cdot f^{2} d V=: \Lambda_{k}^{*} \cdot \int_{C}\langle A\rangle^{2} \cdot f^{2} d V
$$

where $C_{H ; A, 0}$ is the $A+B$-Laplacian from Chapter 2.4 .

For the eigenvalue estimate on $S_{C}$ we write for any $\lambda<\Lambda_{k}^{*}$ :

$$
\begin{aligned}
& \int_{S_{C}} f \cdot\left(L_{C, n}\right)_{\lambda} f d V=\int_{S_{C}}|\nabla f|^{2}-\left(\frac{n-2}{4(n-1)} \cdot|A|^{2}+\lambda \cdot\langle A\rangle^{2}\right) \cdot f^{2} d V \geq \\
\geq & \int_{S_{C}}|\nabla f|^{2}-\frac{1}{4} \cdot|A|^{2} \cdot f^{2} d V-\max \left\{0, \lambda / \tau_{k}\right\} \cdot \int_{S_{C}}|\nabla f|^{2}+|A|^{2} \cdot f^{2} d V .
\end{aligned}
$$

For $\lambda / \tau_{k}<1 / 16$ and $|f|_{L^{2}}=1$, this can be refined to

$$
\geq \frac{1}{3} \int_{S_{C}}|\nabla f|^{2}-|A|^{2} \cdot f^{2} d V \geq \frac{1}{3} \mu_{C,\left(J_{C}\right)_{0}^{\times}} \geq-\frac{(k-2)^{2}}{12} .
$$

Hence, for $\lambda_{k}^{*}:=\min \left\{\Lambda_{k}^{*}, 1 / 16\right\} / 2$ we have $\mu_{C,\left(L_{C, n}\right)_{\lambda}^{\times}} \geq-\frac{(k-2)^{2}}{12}$ for $\lambda \leq \lambda_{k}^{*}$.

For $\lambda \in\left(0, \lambda_{k}^{*}\right]$, we observe again, as in Proposition 4.5 , that there is $f \in C_{0}^{1}\left(S_{C} \backslash \Sigma_{S_{C}}\right)$ with

$$
\int_{S_{C}}|\nabla f|^{2}+\left(\left.\frac{n-2}{4(n-1)} \cdot \operatorname{scal}_{C}\right|_{S_{C}}-\left.\lambda \cdot\langle A\rangle\right|_{S_{C}} ^{2}\right) \cdot f^{2} d V<0 .
$$

Similarly as before, there exist constants $\eta_{\lambda}^{*}$ and $\vartheta_{\lambda}^{*}>0$, depending only on $\lambda>0$ and $k$, and such that $\mu_{C,\left(L_{C, n}\right)_{\lambda}^{\times}}^{*}<-\eta_{\lambda}^{*}<0$. Therefore

$$
0>-\vartheta_{\lambda}^{*}>\alpha_{+} \text {and } \alpha_{-}>\vartheta_{\lambda}^{*}-(k-2)>-(k-2)
$$

for any singular area minimizing $k$-dimensional cone $C^{k}$. 
Complementary to the previous discussions where we mostly focussed on the radial growth rate, we now describe some global properties of the spherical component $\psi_{C}(\omega)$ of $\Psi_{ \pm}[n, k](\omega, r)=\psi_{C}(\omega) \cdot r^{\alpha_{ \pm}}$which is defined over $S_{C} \backslash \Sigma_{S_{C}}$. In particular, this will yield uniform estimates for the radial growth near the singular set.

Proposition 4.7 (Global Harnack Estimates for $\left.\psi_{C}\right)$ For any $k$ with $n \geq k \geq 7, \lambda \in\left(0, \lambda_{k}^{*}\right]$ and cone $C^{k} \in \mathcal{S C}_{k}$, we consider the two solutions $\Psi_{ \pm}[n, k](\omega, r)=\psi_{C}(\omega) \cdot r^{\alpha_{ \pm}}$of $\left(L_{C^{k}, n}\right)_{\lambda} \phi=0$ corresponding to 0 resp. $\infty$ in the Martin boundary $\widehat{\sigma}_{C}$. Then there is a constant $a_{n, k, \lambda}>0$ depending on $n, k$ and $\lambda$, and a constant $b_{n, k, \lambda, \eta_{\lambda}^{*}}$ depending on $n, k, \lambda$ and $\rho>0$ defined by $\rho^{2}:=\lambda / \eta_{\lambda}^{*}$ (with $\eta_{\lambda}^{*}$ from Proposition 4.6) such that:

(i) $\left|\psi_{C}\right|_{L^{p}\left(S_{C} \backslash \Sigma_{S_{C}}\right)}<\infty$ for $p<\frac{k-1}{k-3}$.

(ii) $\left|\psi_{C}\right|_{L^{1}\left(S_{C} \backslash \Sigma_{S_{C}}\right)} \leq a_{n, k, \lambda} \cdot \inf _{\omega \in S_{C} \backslash \Sigma_{S_{C}}} \psi_{C}(\omega)$.

(iii) $\sup _{\omega \in \mathbb{E}(\rho)} \psi_{C}(\omega) \leq b_{n, k, \lambda, \rho} \cdot\left|\psi_{C}\right|_{L^{1}\left(S_{C} \backslash \Sigma_{S_{C}}\right)}$, where $\mathbb{E}(\rho)=\left\{x \in S_{C} \mid\langle A\rangle(x) \leq\right.$ $\left.\rho^{-1}\right\}$.

Similarly, if $q<\frac{k}{k-2}$, then $|v|_{L^{q}\left(B_{1}(0) \cap C^{k}\right)}<\infty$ for any solution $v>0$ of $\left(L_{C^{k}, n}\right)_{\lambda} \phi=0$.

Proof For $0<\rho^{2}=\lambda / \eta_{\lambda}^{*}<-\lambda / \mu_{C,\left(L_{C, n}\right)_{\lambda}^{\times}}^{*}$ and any $C \in \mathcal{S} \mathcal{H}_{n}^{\mathbb{R}}$ we have

$$
\frac{n-2}{4(n-1)} \cdot|A|_{C}^{2}(\omega, 1)+\lambda \cdot\left(\langle A\rangle^{\times}\right)^{2}(\omega)+\mu_{C,\left(L_{C, n}\right)_{\lambda}^{\times}}^{*}>0 \text { on } \mathbb{I}(\rho) .
$$

Hence $\psi_{C}(\omega)>0$ is a superharmonic function on $\mathbb{I}(\rho): \psi_{C}$ solves the equation

$-\Delta_{S_{C}} \psi_{C}(\omega)-\left(\frac{n-2}{4(n-1)} \cdot|A|_{C}^{2}(\omega, 1)+\lambda \cdot\langle A\rangle^{\times}(\omega)^{2}\right) \cdot \psi_{C}(\omega)-\mu_{C,\left(L_{C, n}\right)_{\lambda}^{\times}}^{*} \cdot \psi_{C}(\omega)=0$.

From this the pointwise estimate (34) implies $-\Delta_{S_{C}} \psi_{C}(\omega) \geq 0$ on $\mathbb{I}(\rho)$.

Although $S_{C} \subset \partial B_{1}(0)$ is not a global area minimizer, it is an almost minimizer and it shares the regularity theory with proper area minimizers. We can also locally apply the Bombieri-Giusti Harnack inequality [5, Theorem 6 p. 39] in the following form: For any superharmonic function $w>0$ defined on the regular region of $B_{R}(x) \cap S_{C}$ for a sufficiently small extrinsically measured radius $R>0$, we have

$$
0<\left\{\frac{1}{\operatorname{Vol}_{n-1}\left(S_{C} \cap B_{r}(x)\right)} \int w^{p}\right\}^{1 / p} \leqq C \cdot \inf _{B_{r}(x)} w
$$

for $r \leqq \beta_{n} \cdot R$ and $p<\frac{k-1}{k-3}$ with constants $C=C\left(S_{C}, p\right)$ and $\beta_{n}>0$. We apply this to a finite cover $B_{r}\left(p_{j}\right), j=1, \ldots m$, of $\Sigma_{S_{C}}$ by sufficiently small balls with $B_{R}\left(p_{j}\right) \subset \mathbb{I}(\rho)$.

Since the complement in $S_{C} \backslash \Sigma_{S_{C}}$ of these open balls is compact, $\inf _{S_{C} \backslash \Sigma_{S_{C}}} \psi_{C}(\omega)>0$ and $\left|\psi_{C}\right|_{L^{p}\left(S_{C} \backslash \Sigma_{S_{C}}\right)}<\infty$. Moreover, we obtain for each individual cone $C$ the (trivial) estimate $\sup _{\omega \in \mathbb{E}(\rho)} \psi_{C}(\omega) \leq b \cdot\left|\psi_{C}\right|_{L^{1}\left(S_{C} \backslash \Sigma_{S_{C}}\right)}$ for some suitably large $b=b_{C, n, k, \lambda, \rho}>0$. Since the $\psi_{C}$ are unique up to a multiple, the compactness of $\mathcal{S C}_{k}$, the naturality of $|A|$ and $\langle A\rangle$, and the standard elliptic theory for $\left(L_{C, n}\right)_{\lambda}^{\times}$imply for all $C^{k} \in \mathcal{S C}_{k}$ the existence of some common $a_{n, k, \lambda}>0$ such that

$$
\left|\psi_{C}\right|_{L^{1}\left(S_{C} \backslash \Sigma_{S_{C}}\right)} \leq a_{n, k, \lambda} \cdot \inf _{\omega \in S_{C} \backslash \Sigma_{S_{C}}} \psi_{C}(\omega) .
$$

In this way we also get a common $b_{n, k, \lambda, \rho}>0$ for all cones such that $\sup _{\omega \in \mathbb{E}(\rho)} \psi_{C}(\omega) \leq$ $b_{n, k, \lambda, \rho} \cdot\left|\psi_{C}\right|_{L^{1}\left(S_{C} \backslash \Sigma_{S_{C}}\right)}$. 
The assertion that $|v|_{L^{q}\left(B_{1}(0) \cap C^{k}\right)}<\infty$ for $q<\frac{k}{k-2}$ and a solution $v>0$ of $\left(L_{C^{k}, n}\right)_{\lambda} \phi=0$ follows completely similarly by invoking again the Bombieri-Giusti Harnack inequality.

Funding Information Open Access funding provided by Projekt DEAL.

Open Access This article is licensed under a Creative Commons Attribution 4.0 International License, which permits use, sharing, adaptation, distribution and reproduction in any medium or format, as long as you give appropriate credit to the original author(s) and the source, provide a link to the Creative Commons licence, and indicate if changes were made. The images or other third party material in this article are included in the article's Creative Commons licence, unless indicated otherwise in a credit line to the material. If material is not included in the article's Creative Commons licence and your intended use is not permitted by statutory regulation or exceeds the permitted use, you will need to obtain permission directly from the copyright holder. To view a copy of this licence, visit http://creativecommonshorg/licenses/by/4.0/.

\section{References}

1. Ancona, A.: On positive harmonic functions in cones and cylinders. Rev. Mat. Iberoam. 28, 201-230 (2012)

2. Balinsky, A., Evans, D., Lewis, R.: The Analysis and Geometry of Hardy's Inequality. Springer (2015)

3. Barozzi, E., Gonzalez, E., Massari, U.: On the generalized mean curvature. Calc. Var. 39, 491-523 (2010)

4. Bers, L., John, F., Schechter, M.: Partial Differential Equations. Wiley \& Sons (1964)

5. Bombieri, E., Giusti, E.: Harnack's inequality for elliptic differential equations on minimal surfaces. Invent. Math. 15, 24-46 (1972)

6. Bonk, M., Heinonen, J., Koskela, P.: Uniformizing Gromov hyperbolic spaces, Astérisque. pp 270 (2001)

7. Evans, L.: Partial differential equations, graduate studies in mathematics 19 AMS (2010)

8. Eichmair, M.: The Plateau problem for marginally outer trapped surfaces. J. Diff. Geom. 83, 551-583 (2009)

9. Fuchs, M.: Topics in the Calculus of Variations. Advanced Lectures in Mathematics, Vieweg (1994)

10. Giusti, E.: Minimal Surfaces and functions of bounded variations. Birkhäuser (1984)

11. Giaquinta, M., Modica, G., Sourek, J.: Cartesian Currents in the Calculus of Variations. vol. I, Springer (1998)

12. Gilbarg, D., Trudinger, N.: Elliptic partial differential equations of second order. Springer (1983)

13. Ghoussoub, N., Moradifam, A.: Functional inequalities: new perspectives and new applications, math. Surveys and monographs 187 AMS (2013)

14. Gromov, M.: Positive curvature, macroscopic dimension, spectral gaps and higher signatures, Functional Analysis on the eve of the 21st century. vol. 2, Progr. Math. 132, Birkhäuser, pp 1-213 (1996)

15. Helffer, B.: Spectral Theory and Its Applications. Cambridge Univ.Press, Cambridge Studies in Adv. Math. (2013)

16. Kazdan, J., Warner, F.: Existence and conformal deformations of metrics with prescribed gaussian and scalar curvature. Ann. Math. 101, 317-331 (1975)

17. Lohkamp, J.: Potential theory on minimal hypersurfaces i: singularities as martin boundaries. Potential Analysis, pp. 1-36. https://doi.org/10.1007/s11118-019-09815-6 (2019)

18. Lohkamp, J.: Hyperbolic unfoldings of minimal hypersurfaces. Analysis and Geometry in Metric Spaces 6, 96-128 (2018). https://doi.org/10.1515/agms-2018-0006

19. Mars, M.: Stability of marginally outer trapped surfaces and applications, in recent trends in lorentzian geometry, springer proc. in mathematics and statistics 26, pp. 110-138. Springer, New York (2013)

20. Simon, L.: Lectures on geometric measure theory, proceedings of the centre for mathematical analysis, australian national university canberra (1983)

21. Schoen, R., Yau, S.T.: Existence of incompressible minimal surfaces and the topology of three dimensional manifolds with non-negative scalar curvature. Ann. Math. 110, 127-142 (1979) 
22. Tamanini, I.: Boundaries of Cacciopoli sets with hölder continuous normal vector. J. Reine Angew. Math. 334, 27-39 (1982)

23. Tamanini, I.: Regularity Results for Almost Minimal Oriented Hypersurfaces in $\mathbb{R}^{n}$. Quaderni del Dipartimento di Matematica dell Università di Lecce, Università di Lecce (1984)

24. Zeidler, E.: Applied Functional Analysis. Applications to Mathematical Physics, Springer (1995)

Publisher's Note Springer Nature remains neutral with regard to jurisdictional claims in published maps and institutional affiliations. 